\title{
Le manuel scolaire : une ressource au «statut paradoxal »
}

Rapport de l'enseignant au manuel scolaire de mathématiques à l'école élémentaire

The textbook: a resource with a " paradoxical status »

Elementary school teachers' relationship to the mathematical textbook

\section{Maryvonne Priolet et Éric Mounier}

\section{OpenEdition \\ Journals}

Édition électronique

URL : https://journals.openedition.org/educationdidactique/3041

DOI : 10.4000/educationdidactique.3041

ISSN : 2111-4838

\section{Éditeur}

Presses universitaires de Rennes

\section{Édition imprimée}

Date de publication : 28 août 2018

Pagination : $79-100$

ISBN : 978-2-7535-7554-7

ISSN : 1956-3485

Référence électronique

Maryvonne Priolet et Éric Mounier, « Le manuel scolaire : une ressource au «statut paradoxal » », Éducation et didactique [En ligne], 12-1 | 2018, mis en ligne le 28 août 2020, consulté le 16 août 2022 URL : http://journals.openedition.org/educationdidactique/3041 ; DOI : https://doi.org/10.4000/ educationdidactique.3041 


\title{
LE MANUEL SCOLAIRE : UNE RESSOURCE AU «STATUT PARADOXAL » RAPPORT DE L'ENSEIGNANT AU MANUEL SCOLAIRE DE MATHÉMATIQUES À L'ÉCOLE ÉLÉMENTAIRE
}

\author{
Maryvonne Priolet \\ ICARE (EA 7389), université de la Réunion, CUFR de Mayotte \\ Éric Mounier \\ Université Paris-Est, Laboratoire de Didactique André Revuz (LDAR-EA 4434), \\ UPD, UPEC, UCP, université de Rouen, université d'Artois
}

Près de quarante années après la thèse d'État de Jacques Priouret (1977), la recherche présentée dans cet article repose la question de l'usage du manuel scolaire par les enseignants de l'école élémentaire en France. Face aux constats de "profusion » et de " diversité » de l'offre éditoriale concernant les manuels de mathématiques parus entre 2008 et 2015 pour l'école élémentaire, les auteurs de cet article s'interrogent sur la place occupée par ces ressources dans les classes ainsi que sur l'utilisation des guides pédagogiques par les professeurs. Les observations directes et les analyses effectuées dans dix classes de $\mathrm{CMl}$ conduisent à dégager un double paradoxe et ainsi à revenir sur le « statut paradoxal » du manuel initialement destiné aux élèves.

Mots-clés : manuels scolaires, mathématiques, école élémentaire en France, pratiques enseignantes, offre éditoriale.

The textbook: a resource with a "paradoxical status». Elementary school teachers' relationship to the mathematical textbook

About forty years after Jacques Priouret's thesis (1977), this article presents a research based on the question of the use of textbooks by teachers at the primary school in France. Because of reports of "profusion" and "diversity" of the editorial offer concerning the mathematical textbooks printed between 2008 and 2015, the authors query the place occupied by these textbooks in lessons as well as the use of educational guides by teachers. The direct observations and analyses made in ten classes lead the authors to highlight a double paradox and thus to raise the "paradoxical status" of the textbook initially intended for the pupils.

Keywords: mathematical textbooks, mathematics, French primary school, teaching practices, editorial offer. 


\section{INTRODUCTION}

Près de deux cents ans après son entrée massive dans les écoles et à l'heure de l'accès à des ressources numériques, le manuel scolaire est toujours l'objet d'une littérature scientifique abondante. Depuis une vingtaine d'années, se sont développés des travaux de recherche centrés sur son usage dans les classes de l'école primaire.

Des études prennent appui sur le contexte du Canada (Spallanzani et al., 2001 ; Nicol \& Crespo, 2006 ; Lebrun, 2006 ; Lenoir et al., 2007). D’autres, centrées sur le contexte de la France, concernent parfois l'usage du manuel dans différentes disciplines enseignées à l'école primaire (Priouret, 1977 ; Métoudi \& Duchauffour, 2001) ou se centrent sur un domaine spécifique tel que celui des mathématiques avec soit un même manuel-cible (Arditi, 2011), soit une population-cible tels que des professeurs des écoles « expérimentés et sans lien avec la recherche " (Margolinas \& Wozniak, 2009a, p. 65), ou des professeurs des écoles maîtres-formateurs (Priolet, 2014). D'autres études s'intéressent à l'usage du manuel dans d'autres niveaux d'enseignement, au collège par exemple (Gueudet \& Trouche, 2010). Mais l'abondance de travaux ne signifie pas pour autant l'existence d'un statut privilégié du manuel scolaire dans les classes. Souvent décrié à l'instar du titre «Plus de manuels scolaires! (Freinet, 1928), le manuel scolaire a été jusqu'à inspirer " un sentiment de mépris à ceux qui faisaient profession de traiter la pédagogie dans un registre élevé » (Priouret, 1977 , p. 817). Le présent article interroge la place du manuel scolaire de mathématiques dans l'activité enseignante de professeurs des écoles en France en ce début du XXI ${ }^{\mathrm{e}}$ siècle. Il s'appuie sur l'étude empirique que nous avons réalisée à la demande du Conseil national d'évaluation du système scolaire $(\mathrm{CNESCO})^{1}$ (Mounier \& Priolet, 2015); il concerne l'activité de professeurs ordinaires ayant de deux à quinze années d'ancienneté d'enseignement et disposant dans leur classe de manuels scolaires.

La problématique de notre recherche, centrée sur la question de l'usage, de nos jours, par des professeurs des écoles, de manuels de mathématiques et de guides pédagogiques, sera exposée dans la première partie de cet article. Le cadre de référence sera présenté dans la deuxième partie, sachant que nous considérons l'activité enseignante au sens de Amigues (2003) pour analyser le type d'usage par le professeur, à la fois pour concevoir son enseignement, en dehors de la classe, et pour mettre en œuvre cet enseignement, dans la classe, en présence des élèves. Après avoir défini ce que nous entendons par « manuel scolaire », par « guide pédagogique », par « ressource », nous nous tournerons vers les travaux de Leroyer (2013) afin de questionner le rapport de l'enseignant aux ressources dans le contexte de la conception de son enseignement. Les travaux de Rey (2001) sur les dispositifs didactiques, ceux de Margolinas et Wozniak (2009a), de Priolet (2014) et d'Arditi (2011) seront mobilisés pour considérer le rapport au support dans l'activité d'enseignement. La méthodologie employée sera présentée en troisième partie avec, pour le recueil de données, le recours à la fois à l'observation et aux entretiens semi-directifs ; le traitement des données s'appuiera sur une « analyse de contenu » (Bardin, 2007). Nous poursuivrons par la présentation des résultats, en quatrième partie, en caractérisant la place du manuel de mathématiques dans l'activité enseignante puis en comparant les pratiques de deux professeurs quant à l'utilisation d'un même manuel dans le cadre d'une même séquence de mathématiques en classe de CM1, et enfin en considérant la place du manuel dans la formation des enseignants. Avant de conclure, la cinquième partie sera réservée à l'analyse et à l'interprétation de ces résultats.

\section{ProblémATIQUe}

Dans le système éducatif français, l'enseignant dispose de la liberté pédagogique ${ }^{2}$ et peut ainsi décider d'interagir dans sa pratique professionnelle avec les ressources ${ }^{3}$ de son choix, autrement dit avec tout ce qui est susceptible de lui permettre de se « re-sourcer », en référence au verbe employé par Adler, Reed, Lelliott et Setati (2002, p. 54). Se pose alors la question du travail documentaire au sens de " processus articulant étroitement conception et mise en œuvre » (Gueudet \& Trouche, 2010, p. 58), effectué par les professeurs. À l'heure où « la maîtrise des technologies de l'information et de la communication et le bon usage des ressources numériques, notamment pédagogiques, constituent un enjeu et une opportunité majeurs en matière éducative ${ }^{4}$ ", on peut se demander quelle est la place réservée actuellement dans les classes au manuel scolaire, cette ressource matérielle (Adler, 2010, p. 28) initialement dénom- 
mée livre élémentaire par Condorcet (1792) dans son Discours à l'Assemblée nationale. Cette ressource « dont le marché de masse a véritablement débuté en 1833 avec le vote de la loi Guizot » (Mollier, 2002, p. 128) a fait l'objet de plusieurs études.

Dans le contexte institutionnel, les publications liées aux manuels scolaires et à leurs usages dans les classes sont assez peu nombreuses. On peut citer l'enquête réalisée par Chaillet ${ }^{5}$ (1985), ou celle conduite par Métoudi et Duchauffour (2001) à la demande de l'Institut universitaire de formation des maîtres (IUFM) de Paris et de l'association SAVOIR LIVRE, ainsi que plusieurs rapports de l'Inspection générale (IGEN, 1998, 2010, 2012). En 2008, les programmes nationaux d'enseignement de l'école élémentaire (MEN, 2008) ${ }^{6}$ mentionnaient seulement que « l'appui sur un manuel de qualité pour chacun des volets de l'enseignement du français est un gage de succès » (p. 19); ceux publiés en 2015 (MEN, 2015) ${ }^{7}$ n'évoquent pas non plus la question $\mathrm{du}$ recours au manuel scolaire dans le domaine des mathématiques. De la même façon, alors que le référentiel (MEN, 2010) ${ }^{8}$ définissant les compétences à acquérir par les professeurs des écoles indiquait clairement que le professeur était conduit « à apprécier la qualité des documents pédagogiques (manuels scolaires numériques ou non et livres du professeur associés, ressources documentaires numériques ou non, logiciels d'enseignement, etc. $)^{9} "$, le référentiel actuel (MEN, 2013) ${ }^{10}$ ne comporte plus de référence explicite au manuel scolaire, même si on peut toutefois considérer que celle-ci est implicite lorsque sont évoquées les « ressources disponibles» dans la compétence invitant les professeurs à s'engager dans une démarche de développement professionnel. Est-ce à dire que le manuel scolaire tendrait à disparaître des classes ? Ou faut-il considérer que la «panoplie de manuels» (Priouret, 1977, p. 818) dont disposaient les enseignants dans les années soixante-dix tendrait à s'amenuiser ?

L'étude que nous avons conduite à la demande du CNESCO (Mounier \& Priolet, 2015), à partir de l'inventaire ${ }^{11}$ des manuels scolaires de mathématiques parus de mai 2008 à janvier 2015 à destination des élèves de l'école élémentaire, révèle une profusion et une diversité d'ouvrages sur le marché de l'édition scolaire avec la présence de plus de 120 titres $^{12}$ répartis en 26 collections différentes issues de 12 maisons d'édition (Annexe 1). Toutefois, dans cet ensemble documentaire, chaque manuel ne s'adresse qu'à un seul niveau d'enseignement, du CP au CM2. Il n'existe ainsi, à notre connaissance, aucun manuel de mathématiques destiné à une classe multiniveaux, alors qu'en France le pourcentage de ce type de classes est de 47,5\%13. L'unité de référence du manuel est la classe, ce n'est pas le cycle d'apprentissage $^{14}$. Dès lors, on peut se demander, en considérant un niveau d'enseignement donné, quelle place les enseignants accordent à cette ressource.

Le manuel scolaire, en tant qu'objet de recherche, a généré à partir des années 1980, notamment en France et au Québec, plusieurs études analytiques plutôt centrées sur les contenus et sur les aspects idéologiques des manuels (Choppin, 1980 ; Caritey, 1993). Dès les années 1975, dans sa thèse d'État, Priouret (1977) en conduisant une « réflexion sur le statut paradoxal du livre scolaire ${ }^{15}$ » a ouvert la voie à d'autres types de travaux centrés cette fois-ci sur l'utilisation des manuels dans les classes.

Réalisée en France par Métoudi et Duchauffour (2001), une enquête par questionnaire a permis d'étudier le rôle et la place des manuels dans 63 classes de l'école élémentaire pour lesquelles les enseignants avaient choisi un ensemble de manuels dans une panoplie proposée par six maisons d'édition scolaire. S'agissant du domaine des mathématiques, les résultats de cette étude montrent une diversité d'utilisation des manuels de l'élève et des guides pédagogiques choisis par les enseignants. On relève par exemple que 23,5\% des enseignants indiquent avoir utilisé le manuel de l'élève uniquement pour leur usage personnel, et que $22,3 \%$ déclarent avoir utilisé un autre manuel de l'élève en plus du manuel fourni pour l'étude considérée. Les manuels de mathématiques sont déclarés être fréquemment exploités comme recueil d'exercices $(38,0 \%)$ mais très faiblement utilisés comme source documentaire (6,2\%). Quant au guide pédagogique, 36,4\% déclarent le posséder mais ne pas y recourir.

Dans le même temps, au Québec, dans le cadre d'une recherche triennale conduite sur la période 1997-2000 par Spallanzani et al. (2001), l'analyse des 54 réponses à une enquête par questionnaire adressée à des enseignants de troisième cycle du primaire ${ }^{16}$, a permis de dégager quatre conceptions dominantes du rôle des manuels en réponse à la question « Que représentent les manuels pour vous ? » : manuel comme " aide à la planification », comme «source de documents », comme "source d'informations » et comme «source d'exercices» (p. 170). Pour ces 
mêmes enseignants, interrogés cette fois-ci sur le rôle du manuel par rapport aux élèves, le manuel est « à la fois un outil de référence et de consultation, une source d'exercices et un outil de lecture pour l'élève ». Cependant, il n'est pas majoritairement considéré comme « support indispensable pour réaliser les apprentissages » (p. 170-171). Spallanzani et al. (2001) ont également fait ressortir deux principaux profils d'enseignants liés au recours aux manuels scolaires avec d'une part, les enseignants en début de carrière qui considèrent le manuel comme « source d'activités d'apprentissage » et d'autre part les enseignants chevronnés ${ }^{17}$ pour lesquels le manuel est essentiellement « source d'activités et d'instruments d'évaluation $»$ (p. 182-183).

Basée elle aussi sur des données de type déclaratif mais recueillies lors d'entretiens semi-directifs, l'étude conduite par Margolinas et Wozniak (2009a) auprès de dix professeurs des écoles expérimentés, permet d'identifier pour neuf d'entre eux la présence d'un « document générateur ", document qui «porte en lui le germe de l'œuvre» (p. 69). En effet, parmi une diversité de documents utilisés par l'enseignant, « il en est un qui semble à l'origine de cette œuvre » (ibid.).

C'est autour de cette ressource que le professeur conçoit son enseignement. Ce document générateur ${ }^{18}$ est alors source de développement professionnel pour ces professeurs non spécialistes des mathématiques. Réalisée par Priolet (2014) et prenant appui cette fois-ci sur les observations directes des pratiques d'enseignement de neuf professeurs des écoles maîtres formateurs (PEMF) et d'un professeur des écoles débutant, complétées par des entretiens d'autoconfrontation, une autre étude révèle que l'usage du manuel scolaire qu'il soit ou non déclaré " usage pas à pas », inclut, chez les PEMF des ajustements par rapport aux préconisations des auteurs mais que ces ajustements opérés ne vont pas à l'encontre des objectifs généraux de la séquence d'enseignement-apprentissage. Dès lors, la réussite de ces ajustements peut être en grande partie attribuée à l'expertise de ces professeurs qui « tiennent la classe » (Goigoux, Ria, \& Toczek-Capelle, 2010). Par contraste, le professeur des écoles débutant se trouve à un moment donné en décalage avec les consignes des auteurs et risque de ne pas réussir à «maintenir le cap de la séance ». Ces éléments conduisent à interroger le contenu de la formation des enseignants.
En s'appuyant sur l'utilisation du même manuel « Euro Maths CM2 » par cinq enseignants expérimentés, Arditi (2011) montre la variabilité des pratiques des enseignants dans la réalisation d'une même séance issue de ce manuel écrit par des didacticiens des mathématiques. Se pose alors la question des liens entre l'enseignant et les ressources qu'il utilise. Leroyer (2013, p. 148) désigne par « rapport au support» « l'ensemble de ces relations liées aux supports d'enseignement édités qu'un enseignant, considéré comme individu, entretient avec les contraintes et les ressources du contexte professionnel dans lequel il évolue ainsi que celles liées aux formes communes de cette vie professionnelle».

Les différentes recherches ayant pour objet l'utilisation du manuel scolaire et du guide pédagogique par les enseignants s'orientent de plus en plus vers l'observation des pratiques effectives. Toutefois, celles-ci sont souvent circonscrites à un public spécifique tels que les professeurs expérimentés (Margolinas \& Wozniak, 2009a ; Priolet, 2014), ou à un contexte particulier tel que le Québec (Spallanzani et al., 2001) ou à une ressource bien identifiée (Arditi, 2011). On peut se demander, dans le contexte éducatif français, quelle place, à l'heure actuelle, les professeurs ordinaires ${ }^{19}$ accordent effectivement aux différents manuels scolaires de mathématiques présents sur le marché et aux guides pédagogiques associés. En particulier, quel est le type d'usage par le professeur pour concevoir et pour mettre en œuvre son enseignement? En d'autres termes, quel est le rapport au support que les enseignants entretiennent avec ces ressources dans leur activité enseignante?

\section{CADRE DE RÉFÉRENCE}

\section{L'activité enseignante}

Le professeur doit à la fois concevoir et mettre en œuvre son enseignement. L'activité enseignante comprend trois facettes : "préparer la classe ", « prendre la classe » et « faire la classe » (Amigues, 2003, p. 11). Cette activité résulte d'un compromis entre les objectifs de l'enseignant, ses propres buts, ses contraintes et les ressources de son milieu de travail (Goigoux, 2007, p. 47). Elle dépasse le seul cadre de la salle de classe et s'inscrit, ou du moins devrait s'inscrire, dans une dimension collective 
puisque, en règle générale, le professeur appartient à une équipe d'enseignants nommés dans une même école. Il s'agit donc de considérer l'activité enseignante hors la classe et dans la classe. À l'instar de Goigoux (2002), nous réservons l'expression « activité d'enseignement » à l'activité du professeur en présence de ses élèves et en situation d'enseignement.

\section{Ressources, manuel scolaire et guide pédagogique}

Dans le cadre de son activité enseignante, le professeur interagit avec un ensemble de ressources. Pour définir le concept de ressource « dans et pour l'éducation ", Adler (2010, p. 25) renvoie à deux acceptions : une "réserve " dans laquelle le professeur peut puiser, et l'action de «nourrir à nouveau », différemment, conduisant ainsi au verbe « re-sourcer ». Nous considérons la ressource comme « produit de l'activité humaine, élaboré pour s'inscrire dans une activité finalisée » (Rabardel, 1995, cité dans Gueudet \& Trouche, 2010, p. 58). En tant que produit d'un auteur, le manuel scolaire et le guide pédagogique, support d'enseignement ou support pour la conception de la séance, constituent des ressources pour l'enseignant.

Pour caractériser la ressource " Manuel scolaire », nous nous référons à la définition pragmatique choisie par le groupe d'Inspecteurs généraux rédacteur d'un rapport spécifique sur cette ressource (IGEN, 1998). Nous considérons ainsi comme « manuel scolaire tout support pédagogique (livres ou fiches) qui doit être acquis par l'élève (lycée) ou qui est mis à sa disposition par l'établissement (école primaire et collège) ». Par extension, notre étude concerne tout ouvrage relié ou broché susceptible d'être mis en classe à portée de main des élèves et conçu en relation avec les programmes d'enseignement.

Les ressources parfois dénommées « livre du professeur », " guide de l'enseignant », « fichierressources », « livre du maître », " guide du maître », permettent de « mieux comprendre les transactions des professeurs avec les ressources curriculaires en mathématiques »(Remillard, 2010, p. 201). Nous retenons l'expression " guide pédagogique » pour désigner cette documentation annexée au manuel scolaire et destinée au professeur.

\section{Le rapport de l'enseignant aux ressources}

\section{Dans le travail de conception de l'enseignement}

Pour préparer sa classe, le professeur « interagit avec des ensembles de ressources » (Gueudet $\&$ Trouche, 2010, p. 58) qu'il sélectionne, trie, transforme, réorganise en vue de les mettre en ouvre en situation d'enseignement. Leroyer (2013, p. 158) propose une typologie des enseignants en fonction de leur rapport aux ressources utilisées lors de la préparation de la classe : « concepteur, adaptateur, utilisateur ». L'enseignant concepteur élabore ses outils tels que programmations ou supports d'enseignement, à partir de ses connaissances, de son expérience, de sa formation ou de ses lectures ou de ressources existantes. L'enseignant adaptateur sélectionne, modifie, combine, complète les ressources existantes. L'enseignant utilisateur fait confiance aux auteurs de ces ressources.

\section{Dans l'activité d'enseignement}

En référence à la théorie de l'action conjointe en didactique (Sensevy, 2011) selon laquelle le travail du professeur et des élèves peut se concevoir sous la description de deux moments articulés : l'activité didactique in situ au sein de laquelle le professeur fait jouer le jeu didactique et la préparation de cette activité lorsque le professeur construit le jeu qu'il va mettre en œuvre, on peut considérer que le manuel scolaire se situe au carrefour entre la conception de la séance et sa réalisation.

Or, les enseignants se positionnent différemment par rapport à l'utilisation des ressources selon des «modes d'engagement " (Remillard, 2010, p. 214) pouvant être façonnés par des attentes particulières, des convictions, des habitudes ou des expériences passées. Ce positionnement peut avoir un effet important lorsque l'utilisation de la ressource conduit à l'adoption d'un dispositif didactique structurant la séance. Selon Rey, on distingue trois types de dispositifs didactiques dans une classe primaire (Rey, 2001, p. 31-35). Dans le premier dispositif nommé "explication-application », un élément de savoir, par exemple la définition d'un objet mathématique, est présenté à l'élève. Des exercices d'application suivent la présentation de ce savoir. Dans le deuxième dispositif nommé « observation-compré- 
hension-application », il est dans un premier temps demandé à l'élève d'observer un objet, par exemple une figure géométrique puis de généraliser à partir de cette observation. Des exercices d'application sont ensuite proposés. Dans le troisième dispositif nommé "problème-compréhension-application », l'élève, à partir d'une action, au sens de " manipulation d'objets " matériels mais aussi conceptuels, est amené à résoudre un problème (ibid., p. 35). Cette phase est suivie par la mise en forme du savoir puis par une série d'exercices.

Certains enseignants procèdent parfois à des ajustements pouvant, selon leur degré d'expertise et leur niveau de formation, aller jusqu'à conduire à " des problèmes de cohérence entre objets d'enseignements, processus et activités " (Arditi, 2011, p. 361). Par ailleurs, Margolinas et Wozniak (2009a) et Priolet (2014) montrent que, à partir d'un document générateur, des professeurs experts peuvent procéder à des ajustements pertinents tandis que, faute de formation suffisante, les enseignants débutants vont parfois apporter des modifications allant à l'encontre des intentions des auteurs des manuels, spécialistes de didactique.

\section{MÉTHODOLOGIE}

L'étude présentée dans cet article est une étude empirique effectuée selon une approche de type qualitatif sur la base d'un échantillon dit de convenance, c'est-à-dire de type non probabiliste et formé de sujets qui ont consenti librement et volontairement à participer à la recherche dans sa totalité.

\section{Échantillon}

Cet échantillon est constitué de dix professeurs des écoles ayant préalablement déclaré avoir à leur disposition un manuel de mathématiques pour chacun de leurs élèves. Tous les dix sont chargés au moins du niveau $\mathrm{CMl}^{20}$. Le niveau $\mathrm{CMl}$ a été choisi en raison du poids accordé à l'introduction de savoirs nouveaux dans le domaine de l'apprentissage du nombre, avec l'introduction de l'écriture à virgule pour les décimaux. Les dix enseignants, non formateurs, ont une ancienneté générale de service comprise entre deux et quinze années.

Le tableau (Fig. 1) présente les principales caractéristiques de l'échantillon issu de deux académies et de trois départements différents ainsi que la durée des séances durant lesquelles ces enseignants ont été observés en situation d'enseignement dans leur classe.

Figure 1.

Caractéristiques de l'échantillon. Procédures de recueil des données

\begin{tabular}{|l|l|l|l|}
\hline Code école & Acad. & Dép. & $\begin{array}{l}\text { Nb } \\
\text { Classes }\end{array}$ \\
\hline EC-A & Cl-Fd & 63 & 10 \\
\hline EC-B & Cl-Fd & 63 & 3 (RPI) \\
\hline EC-C & Reims & 10 & 5 \\
\hline EC-D & Cl-Fd & 03 & 1 (RPI) \\
\hline EC-E & Cl-Fd & 03 & 6 (ZEP) \\
\hline EC-F & Cl-Fd & 63 & 10 \\
\hline EC-G & Cl-Fd & 03 & 2 (RPI) \\
\hline EC-H & Cl-Fd & 03 & 2 (RPI) \\
\hline EC-I & Cl-Fd & 03 & 6 \\
\hline EC-J & Cl-Fd & 03 & 2 (RPI) \\
\hline
\end{tabular}

\begin{tabular}{|c|c|c|}
\hline $\begin{array}{l}\text { Code } \\
\text { Ens. }\end{array}$ & $\begin{array}{l}\text { Niv } \\
\text { d'ens. }\end{array}$ & AGS \\
\hline A & CM1 & 11 à 15 \\
\hline B & CM1 & 11 à 15 \\
\hline $\mathrm{C}$ & CM1 & 2 à 5 \\
\hline $\mathrm{D}$ & CM1-CM2 & 6 à 10 \\
\hline $\mathrm{E}$ & CM1 & 2 à 5 \\
\hline $\mathrm{F}$ & CE1-CM1 & 11 à 15 \\
\hline G & CE2-CM1 & 6 à 10 \\
\hline $\mathrm{H}$ & CM1-CM2 & 6 à 10 \\
\hline I & CM1 & 6 à 10 \\
\hline $\mathrm{J}$ & CE2-CM1 & 2 à 5 \\
\hline
\end{tabular}

\begin{tabular}{|l|l|l|l|l|}
\hline $\begin{array}{l}\text { Code } \\
\text { Entr. }\end{array}$ & Période & $\begin{array}{l}\text { Durée } \\
\text { Obs. }\end{array}$ & $\begin{array}{l}\text { Durée } \\
\text { Entr. }\end{array}$ & Tr. \\
\hline A & Mars 2015 & $75 \mathrm{~min}$ & $56 \mathrm{~min} 29 \mathrm{~s}$ & $\mathrm{X}$ \\
\hline B & Mars 2015 & $57 \mathrm{~min}$ & $36 \mathrm{~min} 38 \mathrm{~s}$ & $\mathrm{X}$ \\
\hline C & Mars 2015 & $62 \mathrm{~min}$ & $60 \mathrm{~min} 34 \mathrm{~s}$ & $\mathrm{X}$ \\
\hline D & Mars 2015 & $52 \mathrm{~min}$ & $31 \mathrm{~min} 21 \mathrm{~s}$ & $\mathrm{X}$ \\
\hline E & Mars 2015 & $48 \mathrm{~min}$ & $42 \mathrm{~min} 44 \mathrm{~s}$ & $\mathrm{X}$ \\
\hline F & Mars 2015 & $65 \mathrm{~min}$ & $34 \mathrm{~min} 57 \mathrm{~s}$ & $\mathrm{X}$ \\
\hline G & Mars 2015 & $55 \mathrm{~min}$ & $25 \mathrm{~min} 08 \mathrm{~s}$ & $\mathrm{X}$ \\
\hline H & Mars 2015 & $49 \mathrm{~min}$ & $44 \mathrm{~min} 33 \mathrm{~s}$ & $\mathrm{X}$ \\
\hline I & Mars 2015 & $33 \mathrm{~min}$ & $43 \mathrm{~min} 44 \mathrm{~s}$ & $\mathrm{X}$ \\
\hline J & Mars 2015 & $53 \mathrm{~min}$ & $48 \mathrm{~min} 00 \mathrm{~s}$ & Notes \\
\hline
\end{tabular}

Abréviations : Acad. Académie / Cl-Fd: Clermont-Ferrand / Dép. : Département / Nb classes : Nombre de classes de l'école. / Code ens. : Code enseignant / Niv. d'ens. : niveau d'enseignement / AGS : ancienneté générale de service / Code Entr. : Code entretien / Durée Obs. : durée de la séance observée / Durée entr. : durée de l'entretien semi-directif / Tr. : transcription effectuée $(X)$ ou Notes (transcription non effectuée en raison de problèmes techniques d'enregistrement) / RPI : Regroupement Pédagogique Intercommunal / ZEP : Zone d'Éducation Prioritaire. 
Nous avons souhaité dépasser une approche de type quantitatif ou une approche prenant appui sur le seul mode déclaratif des personnes interviewées. L'observation directe dans des classes en présence du chercheur a été retenue pour caractériser les pratiques effectives concernant l'utilisation du manuel de mathématiques par des enseignants du premier degré, donc polyvalents et ainsi pour la plupart non spécialistes des mathématiques. Les séances observées n'ont pas été vidéoscopées, mais des photographies en lien avec l'utilisation du manuel scolaire ou avec celles d'autres artefacts ont été réalisées.

Une grille d'observation de la séance a été établie. Elle comprenait deux parties :

- l'observation de la séance proprement dite avec le repérage des différentes phases de la séance (durée totale, durée de chaque phase), durée des phases d'utilisation du manuel par les élèves, repérage des moments de recours au manuel ou au guide pédagogique par l'enseignant ;

- le recueil de données en lien avec la documentation de l'élève et celle de l'enseignant.

Faisant suite à l'observation des séances, un deuxième mode de recueil de données, composite, s'est articulé autour d'entretiens de type semi-directif. Ces entretiens, prenant souvent appui ponctuellement sur les photographies réalisées au cours de la séance autour de l'usage du manuel par l'enseignant ou par les élèves peuvent être rapprochés, d'un point de vue méthodologique, des entretiens d'autoconfrontation simple (Theureau, 2010). Un guide d'entretien a été élaboré autour des thèmes suivants : préparation de la séance observée, place du manuel dans le déroulement de la séance, choix du manuel, utilisation du guide pédagogique et du manuel de l'élève d'une manière générale et lors de la séance, formation de l'enseignant.

\section{Procédure de traitement des données}

Les données recueillies dans la grille d'observation ont été compilées afin d'extraire celles concernant la présence et la fréquence d'utilisation déclarée du manuel, les ressources utilisées par l'enseignant pour la conception de son enseignement, les durées d'utilisation effective du manuel par l'élève converties en pourcentages de la durée totale de la séance.
Les entretiens ont donné lieu à une transcription intégrale. Pour en saisir la signification, au-delà de la première impression, nous avons opté pour une analyse de contenu (Bardin, 2007). Pour ce faire une première lecture flottante (ibid., p. 126) a été effectuée pour percevoir les contenus dans leur globalité. Des indices ont été choisis en fonction du cadre de référence et des indications ayant émergé lors de notre lecture flottante. Nous avons ensuite procédé à une analyse catégorielle selon les thèmes suivants : séance (phase d'institutionnalisation, obstacles rencontrés par les élèves, ajustements), ressources (manuel de l'élève, guide pédagogique, ressources numériques, autres ressources, fichiers ou manuels), formation (parcours de l'enseignant, rapport de l'enseignant aux mathématiques), conception de l'enseignement (programmation, construction de la séance).

\section{RÉSULTATS}

Dans un contexte où l'édition scolaire propose une large panoplie de manuels de mathématiques, nous rendons compte, dans un premier temps, de la place réservée au manuel par les dix enseignants ordinaires de notre échantillon, dans leur activité enseignante, en repérant d'abord la présence du manuel scolaire dans la salle de classe, puis la place que lui octroie le professeur tant dans l'activité d'enseignement, que lors de la conception de son enseignement. Dans un deuxième temps, nous dégageons quelques résultats de la comparaison de séances de mathématiques avec utilisation d'un même manuel dans le cadre d'une même séquence sur les nombres décimaux. Enfin, nous essayons aussi de déceler la place du manuel dans la formation de ces enseignants.

\section{Place du manuel de mathématiques dans l'activité enseignante}

Dans un premier temps, nous identifions la place donnée par l'enseignant au manuel de mathématiques dans la classe lors des séances d'enseignement. En nous appuyant d'une part sur les déclarations des dix professeurs de notre échantillon et d'autre part sur l'observation de séances, nous nous demandons si le manuel est effectivement mis à portée de main des élèves. Dans un second temps, nous considérons 
la place accordée par le professeur au manuel de l'élève et au guide pédagogique lors de la conception de son enseignement.

\section{Présence du manuel de mathématiques dans la classe à portée de main des élèves}

Dans les dix classes de notre échantillon, un manuel de mathématiques a été acheté par la municipalité pour chaque élève, voire deux manuels dans la classe de l'enseignant $E^{21}$. Dans trois classes, les manuels ne sont pas à portée de main des élèves. Deux enseignants déclarent avoir relégué le manuel sur une étagère ( $C$ et $D$ ). L'enseignant $F$, nouvellement nommé dans l'école, a décidé de ranger aux archives le manuel acheté deux ans auparavant par la municipalité à raison d'un exemplaire par élève. Il « ne le trouve pas du tout adapté à une classe à double niveau $»$.

Au total, sur les onze manuels inventoriés dans les dix classes, on relève cinq titres différents, l'un d'eux « Pour comprendre les mathématiques » étant présent dans cinq classes sur les dix; dans quatre classes les manuels utilisés sont parus antérieurement aux programmes en vigueur ${ }^{22}$, avec une utilisation déclarée très fréquente dans deux de ces classes ( $\mathrm{G}$ et $\mathrm{H}$ ).

Pour autant, la présence d'un manuel (Fig. 2) susceptible d'être mis à disposition de chaque élève n'implique pas une utilisation systématique de cette ressource : les trois enseignants $(C, D$ et $F)$ qui ont relégué le manuel sur une étagère ou aux archives déclarent ne jamais le mettre entre les mains de leurs élèves ${ }^{23}$, deux (A et J) le faire utiliser peu souvent, un
(E) assez souvent, tandis que quatre autres (B, G, H et $\mathrm{I})$ déclarent une utilisation très fréquente.

Quant au choix du manuel, les entretiens révèlent qu'il n'émane pas nécessairement de l'enseignant utilisateur. Seulement deux enseignants (B et H) sur les dix ont eux-mêmes choisi le manuel actuellement présent dans leur classe. Leur choix repose sur :

- le contenu même du manuel : la « batterie d'exercices proposés par rapport au nombre de dessins qui parfois ne servent pas à grandchose ", les indices associés au niveau de difficulté des exercices;

- la concertation au niveau de l'équipe enseignante en vue de la continuité des apprentissages des élèves d'un niveau à l'autre.

Pour les huit autres enseignants sur les dix, le choix a été effectué par leurs collègues qui les ont précédés dans l'école. Il s'en est suivi :

- tantôt une adoption consentie grâce aux exercices (I) et à la programmation que le manuel propose (E), grâce au cadrage qui « évite de se disperser » $(G)$. Enfin, il « évite les photocopies » $(G)$;

- tantôt un rejet total du manuel par l'enseignant actuellement en poste qui va parfois jusqu'à retirer le manuel de la salle de classe pour le placer aux archives (F) ou le reléguer sur une étagère ( $C$ et $\mathrm{D})$;

- tantôt une utilisation épisodique de ce manuel avec adjonction de fiches réalisées par l'enseignant à partir d'un ou deux autres manuels (spécimens) (A et J).

Les motifs évoqués pour la mise à distance totale ou partielle concernent soit le format qui « ne parle pas » à l'enseignant en donnant une « impression de fouillis » (C), soit le contenu avec l'absence ou la non-

Figure 2.

Manuel scolaire : utilisation par l'élève en classe (déclaration des professeurs)

\begin{tabular}{|c|c|c|c|c|c|c|c|c|}
\hline \multirow[b]{2}{*}{ Enseignant } & \multirow[b]{2}{*}{ Niveau } & \multicolumn{3}{|l|}{ Manuel } & \multicolumn{4}{|c|}{$\begin{array}{c}\text { Utilisation par l'élève } \\
\text { (déclaration des professeurs) }\end{array}$} \\
\hline & & Titre & $\begin{array}{c}\text { Année de } \\
\text { parution }\end{array}$ & $\begin{array}{c}\text { Choisi / } \\
\text { Subi }\end{array}$ & Jamais & \begin{tabular}{|c|} 
Peu \\
souvent
\end{tabular} & $\begin{array}{c}\text { Assez } \\
\text { souvent }\end{array}$ & $\begin{array}{c}\text { Très } \\
\text { souvent }\end{array}$ \\
\hline A & CM1 & Le Nouveau Math Elém & \begin{tabular}{|l|}
2001 \\
\end{tabular} & Subi & & $\mathrm{X}$ & & \\
\hline B & CM1 & à portée de Maths & 2012 & Choisi & & & & $\mathrm{x}$ \\
\hline C & CM1 & Pour Comprendre les Maths & 2007 & Subi & $x$ & & & \\
\hline D & CM1-CM2 & Pour Comprendre les Maths & 2008 & Subi & $\mathrm{x}$ & & & \\
\hline \multirow{2}{*}{$E$} & \multirow{2}{*}{ CM1 } & Au rythme des maths & 2011 & Subi & & & $\mathrm{x}$ & \\
\hline & & Outils pour les Maths & 2011 & Subi & & & $\mathrm{x}$ & \\
\hline $\mathrm{F}$ & CE1-CM1 & Pour Comprendre les Maths & 2008 & Subi & $\mathrm{X}$ & & & \\
\hline G & CE2-CM1 & Pour Comprendre les Maths & 2006 & Subi & & & & $\mathrm{X}$ \\
\hline $\mathrm{H}$ & CM1-CM2 & Pour Comprendre les Maths & 2003 & Choisi & & & & $\mathrm{x}$ \\
\hline 1 & CM1 & à portée de Maths & 2011 & Subi & & & & $x$ \\
\hline $\mathrm{J}$ & CE2-CM1 & Pour Comprendre les Maths & 2008 & Subi & & $\mathrm{x}$ & & \\
\hline
\end{tabular}


pertinence ( « contenu trop abstrait ») de certaines leçons ou de mémo (F et D), soit la programmation proposée (D), ou bien encore la difficulté personnelle à " entrer dans » le manuel (C). La structure de la classe est également mise en avant avec la nonadaptation à une classe à plusieurs niveaux en raison notamment $\mathrm{du}$ « flou pour introduire une notion nouvelle» $(\mathrm{F})$.

La question du budget alloué à l'achat des manuels est mentionnée par quatre enseignants (B, $\mathrm{H}, \mathrm{E}$ et $\mathrm{F}$ ) qui précisent qu'ils doivent aussi prendre en compte l'achat de manuels dans les autres disciplines scolaires, évoquant ainsi la polyvalence du métier d'enseignant du premier degré.

\section{Place du manuel dans l'activité d'enseignement observée}

Afin d'identifier les usages que les enseignants font du manuel ${ }^{24}$, voire les ajustements qu'ils y apportent, dix séances de numération ou de calcul en classe de CMl ont été observées : neuf séances sur fractions ou fractions décimales ou nombres à virgule ; une séance sur la division.

$\mathrm{Au}$ cours de l'observation des séances, cinq enseignants (C, D, E, F et J) n'ont à aucun moment demandé aux élèves d'utiliser de manuel. Ces cinq enseignants ont « subi » le manuel déjà présent dans la classe. Pour trois d'entre eux (C, D et F), ce résultat de non-utilisation par les élèves lors de la séance est en concordance avec les déclarations de « rejet total » du manuel présent dans la classe, de même pour l'enseignant J qui déclare une utilisation «peu fréquente $»$.
Quant à l'enseignant E qui déclarait une utilisation assez fréquente des deux manuels déjà présents dans la classe mais adoptés, il précise que pour cette séance portant sur « les fractions dans la vie quotidienne » il a préféré cette année se référer à une séance proposée sur Internet, accompagnée de fiches individuelles permettant de simuler des partages de gâteaux. "J'ai trouvé que la situation de départ permettait une plus grande manipulation, c'était plus concret avec des exemples du quotidien... ".

Lors des cinq autres séances observées, les élèves ont effectivement utilisé à un moment donné un manuel de mathématiques (Fig. 3). Le chronométrage des séances révèle que ce temps d'utilisation ${ }^{25}$ varie entre les séances, d'une part pour la durée d'utilisation ( $25 \%$ de la durée des séances conduites par les enseignants A et B vs $57 \%$ [27\% puis $30 \%$ ]) de la durée de la séance conduite par l'enseignant $G$, d'autre part pour la répartition des temps d'utilisation : une seule phase d'utilisation située dans la deuxième partie de la séance pour deux classes ; une seule phase située en première partie pour l'enseignant $\mathrm{H}$ et deux phases pour l'enseignant $\mathrm{G}$.

Pour l'enseignant $\mathrm{H}$, cette phase d'exercices se situe en début de séance, contrastant ainsi avec les quatre autres séances qui comportent cette phase d'exercice en fin de séance ; pour l'enseignant $G$, la première phase d'utilisation du manuel consiste à lire une situation et à observer afin de découvrir une nouvelle notion : les fractions. À noter que ces deux classes sont des classes à double-niveau : CE2-CM1 et CM1-CM2. Pendant qu'un niveau de classe travaille sous la conduite directe de l'enseignant, l'autre niveau suit les consignes données par le manuel. Toutefois, sur les cinq classes observées en

Figure 3.

Statut déclaré du manuel. Utilisation déclarée et utilisation effective du manuel par les élèves lors des séances observées

\begin{tabular}{|c|c|c|c|c|c|c|c|}
\hline & \multicolumn{2}{|c|}{ Statut du manuel } & \multicolumn{4}{|c|}{ Utilisation déclarée } & \multirow{2}{*}{$\begin{array}{l}\text { Utilisation effective observé e lors d'une sé ance } \\
\text { (en \% du temps total de la sé ance) }\end{array}$} \\
\hline Ens. & Choisi & Subi & Jamais & \begin{tabular}{|c|} 
Peu \\
souvent
\end{tabular} & \begin{tabular}{|c|} 
Asse $z$ \\
souvent
\end{tabular} & \begin{tabular}{|c|} 
Très \\
souvent
\end{tabular} & \\
\hline $\mathrm{A}$ & & Épisodique & & $\mathrm{x}$ & & & $1 \quad 25 \%$ \\
\hline $\mathrm{B}$ & $\bar{x}$ & & & & & $\bar{x}$ & \begin{tabular}{l|l} 
& $25 \%$ \\
\end{tabular} \\
\hline $\mathrm{C}$ & & Rejeté & $\bar{x}$ & & & & \\
\hline $\bar{D}$ & & Rejeté & $\bar{x}$ & & & & \\
\hline \multirow{2}{*}{ E } & & \multirow{2}{*}{ Adopté } & & & $\bar{x}$ & & \\
\hline & & & & & $x$ & & \\
\hline $\mathrm{F}$ & & Rejeté & $x$ & & & & \\
\hline$G$ & & Adopté & & & & $\bar{x}$ & $1 \quad 288$ \\
\hline $\mathrm{H}$ & $\bar{x}$ & & & & & $\bar{x}$ & - 29\% \\
\hline $\mathrm{I}$ & & Adopté & & & & $\bar{x}$ & $\square$ \\
\hline $\mathrm{J}$ & & Épisodique & & $x$ & & & $\square$ \\
\hline
\end{tabular}


double-niveau, D, F et J n'ont pas utilisé le manuel. Il ne semble donc pas y avoir d'effet significatif de la structure de la classe sur l'utilisation du manuel lors des séances observées. Ces observations sont en conformité avec les déclarations des enseignants : D et F déclarent ne « jamais » utiliser le manuel ; J, l'utiliser « peu souvent » tandis que G et $\mathrm{H}$ déclarent l'utiliser « très souvent ».

À l'exception de la première partie de la séance conduite par l'enseignant $G$ dans laquelle le manuel est utilisé pour faire découvrir une situation aux élèves, cette ressource est principalement utilisée comme banque d'exercices. Il est demandé aux élèves d'ouvrir leur manuel à telle page puis d'effectuer individuellement tels exercices. Un temps de correction collective ou individuelle achève la séance, mais dans tous les cas les élèves gardent leur manuel ouvert sur la table, avec un retour à l'énoncé plus ou moins explicite selon les classes et ainsi, un manuel parfois dissimulé en partie sous le cahier.

Dans toutes les classes, les enseignants disposent souvent à portée de main d'un exemplaire du manuel de l'élève ; ils mobilisent aussi d'autres ressources documentaires lors de la préparation des séances.

Au cours des séances observées, les élèves de six classes (B, D, E, G, H, I) ont eu accès à un moment donné de la séance à des jeux mathématiques, le plus souvent fabriqués par l'enseignant à partir de fiches réalisées par d'autres enseignants et extraites d'Internet. Pendant la séance, deux enseignants en début de carrière (moins de 5 années d'ancienneté) ont utilisé, pour l'un un tableau blanc interactif (D) et pour l'autre, un vidéoprojecteur couplé à un ordinateur (J). Cinq autres classes (A, B, C, G et H) utilisent régulièrement des logiciels pour des travaux sur les nombres et notamment sur les nombres décimaux, évoquant l'aide sur écran apportée par la règle graduée aux élèves pour se représenter le partage de l'unité en dixièmes puis en centièmes, ainsi que la nécessité pour les élèves d'utiliser le vocabulaire spécifique pour nommer les nombres décimaux.

\section{Place du manuel et du guide pédagogique lors de la conception globale de l'enseignement}

Parmi les dix enseignants, cinq (A, C, E, H et I) déclarent se référer au guide pédagogique associé au manuel présent dans la classe pour concevoir ou préparer les séances. Mais l'usage du guide n'est jamais exhaustif ; tous les utilisateurs du guide mobilisent d'autres ressources (Fig. 4). Trois enseignants (B, G et J) se réfèrent au manuel de l'élève sans avoir recours à un moment donné au guide de l'enseignant associé à ce manuel. Parmi les quatre professeurs qui déclarent mettre très souvent le manuel entre les mains des élèves, deux mentionnent faire usage du guide pédagogique ( $\mathrm{H}$ et I), les deux autres non (B et $G$ ). Notons en outre que les enseignants $C$ et $F$ qui n'ont pas mis le manuel à portée de main des élèves déclarent l'utiliser pour concevoir leur enseignement. S'agissant des deux enseignants B et H qui ont choisi leur manuel, l'un utilise le guide pédagogique et l'autre ne l'utilise pas.

Les dix enseignants, y compris ceux qui avaient choisi eux-mêmes un manuel et le guide pédagogique associé, citent l'utilisation d'Internet pour préparer leurs séances de mathématiques, laissant ainsi supposer que ce recours aux technologies numériques constitue désormais un passage obligé pour les enseignants lors de la phase de préparation des séances : « J'en trouve pas mal sur Internet, je trouve qu'il y a des collègues qui travaillent très bien et qui font des choses pas mal. » $(\mathrm{H})$.

Figure 4 .

Ressources utilisées par les enseignants pour la conception de l'enseignement

\begin{tabular}{|l|c|c|c|c|c|c|c|}
\hline Ens. & $\begin{array}{c}\text { Le guide } \\
\text { du manuel } \\
\text { de la classe }\end{array}$ & $\begin{array}{c}\text { Autre(s) } \\
\text { guide(s) }\end{array}$ & $\begin{array}{c}\text { Manuel } \\
\text { de } \\
\text { l'élève }\end{array}$ & $\begin{array}{c}\text { Autre(s) } \\
\text { manuel(s) }\end{array}$ & Internet & $\begin{array}{c}\text { Autres } \\
\text { ressources }\end{array}$ & Crée \\
\hline $\mathrm{A}$ & $\mathrm{X}$ & $\mathrm{X}$ & $\mathrm{X}$ & $\mathrm{X}$ & $\mathrm{X}$ & $\mathrm{X}$ & \\
\hline $\mathrm{B}$ & & & $\mathrm{X}$ & $\mathrm{X}$ & $\mathrm{X}$ & $\mathrm{X}$ & \\
\hline $\mathrm{C}$ & $\mathrm{X}$ & & $\mathrm{X}$ & $\mathrm{X}$ & $\mathrm{X}$ & $\mathrm{X}$ & \\
\hline $\mathrm{D}$ & & & & & $\mathrm{X}$ & $\mathrm{X}$ & $\mathrm{X}$ \\
\hline $\mathrm{E}$ & $\mathrm{X}$ & $\mathrm{X}$ & $\mathrm{X}$ & & $\mathrm{X}$ & $\mathrm{X}$ & \\
\hline $\mathrm{F}$ & & $\mathrm{X}$ & $\mathrm{X}$ & & $\mathrm{X}$ & & \\
\hline $\mathrm{G}$ & & & $\mathrm{X}$ & & $\mathrm{X}$ & $\mathrm{X}$ & \\
\hline $\mathrm{H}$ & $\mathrm{X}$ & & $\mathrm{X}$ & & $\mathrm{X}$ & $\mathrm{X}$ & \\
\hline $\mathrm{I}$ & $\mathrm{X}$ & & & & $\mathrm{X}$ & $\mathrm{X}$ & \\
\hline $\mathrm{J}$ & & & $\mathrm{X}$ & & $\mathrm{X}$ & & \\
\hline
\end{tabular}

FIG. 4. - Les "Autres ressources" correspondent à des jeux mathématiques à manipuler fabriqués par l'enseignant ou achetés, des logiciels de géométrie, de problèmes... des documents fournis dans le cadre d'un rallye-mathématique.

Les six utilisateurs (A, C, E, F, H et I) d'un guide pédagogique disent apprécier les programmations ou les progressions proposées qu'ils considèrent comme des repères, allant même jusqu'à nommer ce guide pédagogique " ceinture de sécurité au regard des programmes " (C). Ils sont également en quête d'éléments concernant les séances et le rapport des élèves au savoir mathématique en jeu. Ces ensei- 
gnants trouvent dans ce guide un ensemble structuré, articulé autour de compétences avec une entrée motivante dans le savoir. Quatre d'entre eux (A, E, H et I) lisent avec soin la situation de découverte ou de départ. E apprécie aussi le fait que le guide «pointe les difficultés qu'on peut attendre des élèves ».

L'enseignant B qui n'utilise pas de guide pédagogique souligne son attente d'un plus grand nombre « d'exercices de remédiation». La déclaration de l'enseignant $\mathrm{H}$ « quand j'ai besoin de la séance, je regarde sur le guide téléchargeable qui est offert avec le livre » laisse à penser que la politique de certains éditeurs de mettre en téléchargement gratuit les guides pédagogiques est appréciée par des enseignants.

Mais cet ouvrage, utile pour les uns, rassurant pour les autres comme en témoigne cet enseignant (I), « les maths ce n'est pas ma spécialité loin de là, je me réfère quand même pas mal au guide du maître », est-il pour autant lu et exploité dans son intégralité ? Parmi les six enseignants utilisateurs de guide, trois ( $\mathrm{C}, \mathrm{E}$ et $\mathrm{H})$ déclarent ne pas avoir pris connaissance des intentions liminaires des auteurs. Un autre (A) dit les avoir « simplement survolées", tandis que l'enseignant $F$ indique avoir étudié cet ouvrage avec attention, déclarant même que « c'est extrêmement important », en prenant pour exemple « la technique de la soustraction qu'ils abordent comme cela au CEl où l'on barre le chiffre ».

Place du manuel dans la programmation annuelle, dans le choix de la situation de départ et dans le choix des exercices

Concernant la programmation de l'activité sur l'année, sept enseignants (A, C, E, F, G, H et I) sur les neuf s'étant exprimés sur ce sujet déclarent utiliser celles fournies par le manuel ou le guide pédagogique, les enseignants B « ma programmation, c'est ma sauce à moi » et $\mathrm{D}$ construisent eux-mêmes leur programmation. Il est à noter que le professeur $\mathrm{D}$ a un cursus universitaire en mathématiques.

Les professeurs $C$ et I font confiance au cadre proposé par le manuel ou le guide. Les cinq professeurs (A, E, F, G et H) ont adopté la programmation d'un manuel ou d'un guide mais l'ont ajustée en « espaçant les séances » (A), en changeant la géométrie $(\mathrm{H})$. L'adaptation la plus extrême est celle du professeur A qui a adopté la programmation de
« Math'élém » en début d'année puis a changé de support en cours d'année.

Concernant le choix de la situation de départ, deux enseignants (A et E) font confiance au guide pédagogique et au manuel de l'élève. $\mathrm{B}$ et $\mathrm{D}$, a contrario, n'utilisent pas le guide pédagogique et préfèrent partir de quelque chose de nouveau et construire eux-mêmes leurs situations. C et F simplifient ou modifient les situations proposées. Les professeurs $G, H$ et I portent un regard critique sur les situations proposées, les adoptant si elles leur conviennent mais n'hésitant pas à se référer à d'autres supports si la situation ne leur convient pas. C'est ainsi que G utilise les situations si « elles sont exploitables », que $\mathrm{H}$ n'utilise pas « toutes les situations qui lui paraissent inadaptées à la façon de travailler de ses élèves », et que I « va prendre ailleurs sur Internet quand cela ne lui plaît pas ».

Concernant les exercices, nous avons observé cinq enseignants (A, B, G, H et I) qui utilisaient les exercices du manuel de l'élève lors de la séance observée. Le professeur I ne déclare pas le manuel de l'élève comme ressource utilisée pour la conception de son enseignement dès lors que le guide pédagogique reprend les exercices du manuel. L'enseignant D conçoit la plupart du temps les exercices : montrant l'exercice du jour, il dit « je l'ai construit moi-même ». $\mathrm{C}$, E et $\mathrm{F}$ adaptent une sélection d'exercices trouvés dans une panoplie de manuels ou sur Internet : $C$ écrit « les exercices et problèmes au tableau en les modifiant $» ; E$ « je prends les exercices et parfois je modifie la consigne »; F modifie les valeurs numériques des exercices et leur présentation.

\section{Comparaison des pratiques de deux professeurs relatives à la même séquence d'enseignement}

La comparaison porte sur l'utilisation d'un même manuel et d'un même guide pédagogique par deux enseignants de CMl dans le cadre d'une séquence sur les nombres décimaux portant sur l'introduction de l'écriture à virgule. Dans un premier temps, nous décrivons le contenu et les préconisations présentés dans le guide pédagogique et le manuel scolaire.

Pour les séances numérotées 8 et 9 , le guide pédagogique propose une "piste de recherche» (Fig. 5) qui «pourra se substituer » au «Cherchons ensemble » ou le "précéder ${ }^{26}$. En nous référant au modèle de Rey $(2001)^{27}$, la situation qui 
demande de mesurer différents segments avec une " règle » graduée en dixièmes renvoie à un dispositif didactique du type « observation-compréhensionapplication » (Rey, 2001, p. 32). Pour la « piste de recherche » de la séance 9 , le guide propose à l'enseignant une droite numérique graduée destinée à être tracée au tableau. L'activité consistera alors à faire placer aux élèves plusieurs écritures fractionnaires sur cette droite. À partir de plusieurs exemples, l'enseignant introduira l'écriture à virgule.

Figure 5 .

Extrait du guide pédagogique à portée de maths CM1

$$
\text { (p. 39) }
$$

\section{Piste de recherche}

Travailler au tableau à partir d'une droite numérique graduée.

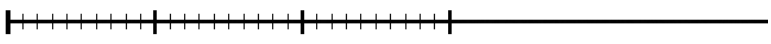

Demander de placer des écritures fractionnaires sur la droite. Prendre un exemple $: \frac{25}{10}$.

Quant au manuel, chaque double page propose sur la partie supérieure de la page de gauche un encart réservé à la rubrique "Cherchons ensemble » (Fig. 6). De par son titre, cette rubrique semble viser à engager les élèves dans un questionnement intellectuel susceptible de conduire à un dispositif didactique du type "problème-compréhension-application » (Rey, 2001, p. 35) basé sur une conception socioconstructiviste de l'apprentissage. Or, la conception même de la page du manuel offre à l'élève la possibilité de se référer directement à des exemples, voire à des conclusions, placées de façon paradoxale à proximité de l'énoncé "Cherchons ensemble ». Dès lors, malgré un titre de rubrique attractif, ces pages du manuel renvoient plutôt à un dispositif didactique basé sur « observation-compréhension-application » (ibid., p. 32). Tout se passe en effet comme si les élèves avaient à observer un objet puis à essayer de le comprendre en vue de s'entraîner à des exercices d'application.
Figure 6.

Partie supérieure de la page 42 (À portée de Maths CM1)

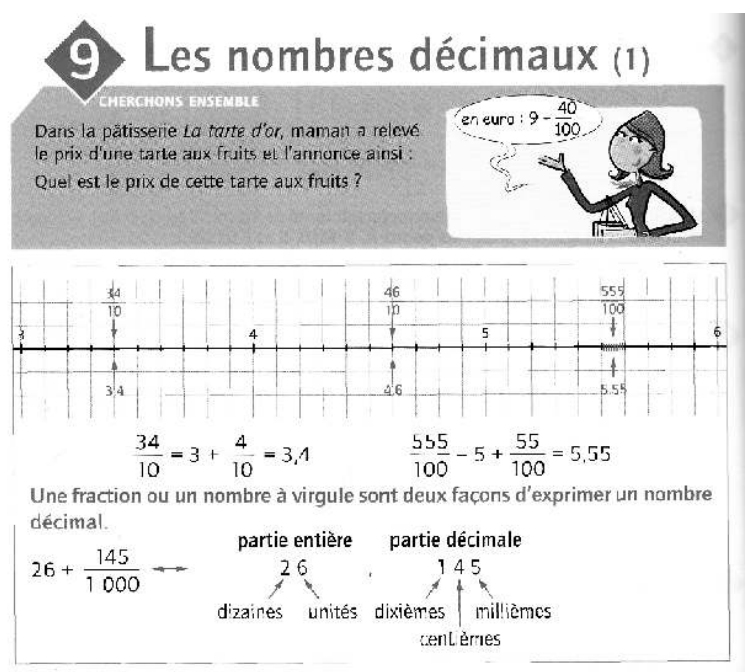

Dans un second temps, nous comparons les séances mises en œuvre par les enseignants B et I. Tandis que dans les deux classes les élèves sont utilisateurs du même manuel ${ }^{28}$, on constate au cours des séances observées que les enseignants B et I ont fait des choix différents pour préparer leur séance et qu'ils ont procédé à des ajustements (Fig. 7).

Figure 7.

Description partielle et éléments de comparaison des séances réalisées par les deux enseignants B et I

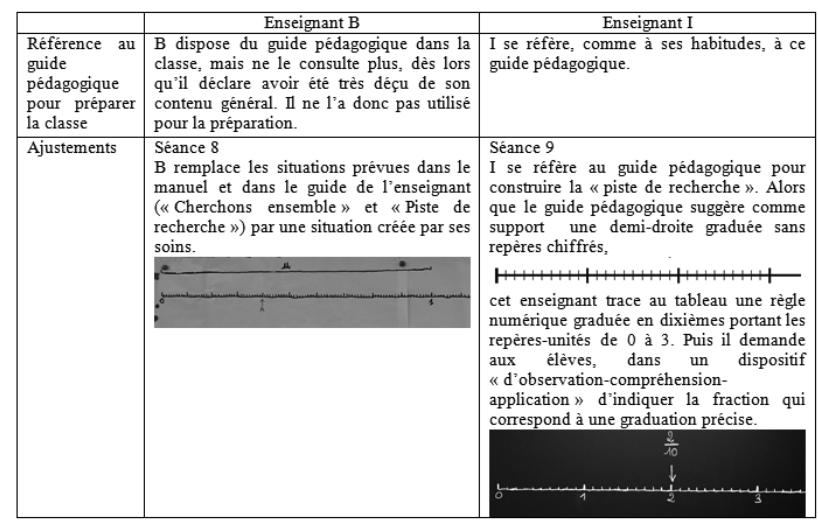

L'enseignant B modifie le dispositif didactique passant d'un modèle « observation-compréhensionapplication » induite par le guide pédagogique à un modèle du type « explication-application».

L'enseignant I modifie le support de la situation de départ en passant d'une demi-droite graduée sans 
repères chiffrés à la droite représentée (Fig. 7). Cette modification de la présentation du support a transformé, en la réduisant, la difficulté de la tâche prévue par les auteurs du manuel.

\section{Place du manuel dans la formation des enseignants}

Parmi les neuf enseignants interrogés sur la formation qu'ils ont pu avoir concernant l'usage du manuel scolaire, trois ( $D, G$ et $H)$ déclarent que cette question n'a jamais été abordée au cours de leur formation initiale ou continue. Notons que ces enseignants sont tous les trois dans la classe 6-10 ans d'ancienneté de service.

Pour les autres, deux catégories peuvent être établies :

- quatre enseignants se rappellent d'un titre de manuel mis en exergue au cours de leur formation initiale : "Ermel » (B et F) et «Cap-Maths » (C et E);

- un autre (A) se remémore les conseils plus globaux qui lui avaient été donnés ;

- d'abord en formation initiale : « À l'IUFM j'avais plutôt l'impression qu'il fallait vraiment que l'on construise nous-mêmes nos situations $»$;

- puis ensuite lors d'une session de formation continue : "On nous a conseillé de ne pas hésiter à nous servir des manuels parce que c'est des gens qui ont réfléchi et qui sont spécialistes dans ces domaines. »

Ces cinq enseignants se situent soit dans la classe 11-15 ans d'ancienneté (A, B et F), soit dans la classe 2-5 ans (C et E).

$\mathrm{Au}$ regard des propos de ces neuf enseignants, on perçoit la diversité des contenus de formation et des points de vue développés par les formateurs. D'après les entretiens, les enseignants considèrent aussi qu'ils sont peu préparés à la question de l'utilisation du manuel scolaire et notamment du manuel de mathématiques.

\section{ANALYSE DES RÉSULTATS}

\section{Manuel scolaire et offre éditoriale}

Le manuel scolaire de mathématiques, en tant qu'ouvrage relié ou broché susceptible d'être mis en classe à portée de main des élèves et conçu en relation avec les programmes d'enseignement, reste bien présent sur le marché de l'édition scolaire avec une offre comptant plus de 120 titres parus depuis la publication des programmes 2008 pour l'école élémentaire jusqu'à janvier 2015. L'offre éditoriale est « foisonnante » rejoignant ainsi un constat effectué une quinzaine d'années auparavant par Métoudi et Duchauffour (2001, p. 17). Elle fait aussi écho à la « panoplie de manuels » (Priouret, 1977, p. 818).

\section{Rapport de l'enseignant au support}

Dès lors que nous considérons l'activité enseignante dans la classe et hors la classe, tant pour l'activité d'enseignement que pour l'activité de conception des séances, nous analysons le rapport au support dans ces deux types d'activité.

\section{Les supports manuel de l'élève et guide de l'enseignant}

La profusion et la diversité des manuels scolaires de mathématiques constatées renvoient nécessairement à la question du choix des manuels par les enseignants utilisateurs (Mounier \& Priolet, 2015, p. 38). Les résultats de notre étude révèlent que ce choix n'incombe pas nécessairement à l'enseignant de la classe puisque la durée de présence d'un manuel dans une école dépasse parfois largement la durée de présence de l'enseignant dans l'école. Polyvalent, donc amené à choisir des manuels dans d'autres disciplines avec un budget parfois limité, l'enseignant reprend alors le manuel de mathématiques présent depuis plusieurs années dans la classe, manuel qu'il n'a donc pas nécessairement choisi et dont il ne sollicite pas le renouvellement en dépit de l'éventuelle obsolescence. C'est ainsi que l'on trouve dans certaines classes des manuels de mathématiques dont la parution est antérieure aux programmes en vigueur, mais aussi des manuels qui, bien que récents, se sont vu relégués aux archives ; comme l'écrivaient Métoudi et Duchauffour (2001, p. 17) «il faut vivre avec ce livre, réussir à bien enseigner avec lui, il est donc essentiel de ne pas se tromper en le sélectionnant ». Le manuel, c'est un compagnon. Et comment faire pour « vivre avec » lui, s'il n'a pas été choisi, comme c'est le cas pour 8 enseignants sur 10 
de notre panel ? Analyser le rapport du professeur au manuel de l'élève et au guide de l'enseignant impose d'abord de s'intéresser au choix de ces supports et à leur utilisation. Quel usage est-il fait de ces deux types de support ? Le tableau suivant (Fig. 8) met en perspective certaines des informations déjà présentées qui peuvent éclairer ce questionnement ${ }^{29}$.

Figure 8 .

Utilisation déclarée de guides et de manuels dans la classe et pour la conception des séances

\begin{tabular}{|c|c|c|c|c|c|c|}
\hline \multirow[b]{2}{*}{$\begin{array}{c}\text { AGS } \\
\text { (en années) }\end{array}$} & \multirow[b]{2}{*}{ Ens. } & \multirow[b]{2}{*}{$\begin{array}{c}\text { Manuel } \\
\text { Subi (S) } \\
\text { ou } \\
\text { Choisi (C) }\end{array}$} & \multirow[b]{2}{*}{$\begin{array}{c}\text { Manuel mis } \\
\text { entre les mains } \\
\text { des élèves }\end{array}$} & \multicolumn{3}{|c|}{ Conception des séances } \\
\hline & & & & $\begin{array}{c}\text { Programmation } \\
\text { établie à partir du } \\
\text { guide et/ou du } \\
\text { manuel } \\
\text { (avec ajustements : } \\
\text { oui, Ajust.) }\end{array}$ & $\begin{array}{c}\text { Ouvrage } \\
\text { utilisé pour } \\
\text { concevoir } \\
\text { les séances }\end{array}$ & $\begin{array}{c}\text { Autres guides } \\
\text { et/ou } \\
\text { manuels) }\end{array}$ \\
\hline 2 à 5 & C & $\mathbf{s}$ & Jamais & Oui & $\begin{array}{c}\text { Guide et } \\
\text { Manuel }\end{array}$ & Manuel \\
\hline 2 à 5 & E & $\mathrm{s}$ & $\begin{array}{c}\text { Assez } \\
\text { souvent }\end{array}$ & Oui, Ajust. & $\begin{array}{c}\text { Guide et } \\
\text { Manuel }\end{array}$ & Guide \\
\hline 2 à 5 & $\mathbf{J}$ & s & $\begin{array}{c}\text { Peu } \\
\text { souvent }\end{array}$ & ? & Manuel & \\
\hline 6 à 10 & D & $\mathrm{s}$ & Jamais & Non & & $\begin{array}{c}\text { Guide et } \\
\text { manuel }\end{array}$ \\
\hline 6 à 10 & G & $\mathbf{s}$ & $\begin{array}{c}\begin{array}{c}\text { Très } \\
\text { souvent }\end{array} \\
\end{array}$ & Oui, Ajust. & Manuel & \\
\hline 6 à 10 & H & C & $\begin{array}{c}\begin{array}{c}\text { Très } \\
\text { souvent }\end{array} \\
\end{array}$ & Oui, Ajust. & $\begin{array}{c}\text { Guide et } \\
\text { Manuel }\end{array}$ & \\
\hline 6 à 10 & I & $\mathrm{s}$ & $\begin{array}{c}\text { Très } \\
\text { souvent }\end{array}$ & Oui & Guide & \\
\hline 11 à 15 & A & $\mathrm{s}$ & $\begin{array}{c}\begin{array}{c}\text { Peu } \\
\text { souvent }\end{array} \\
\end{array}$ & Oui, Ajust. & $\begin{array}{c}\text { Guide et } \\
\text { Manuel }\end{array}$ & $\begin{array}{c}\text { Guide et } \\
\text { manuel }\end{array}$ \\
\hline 11 à 15 & B & C & $\begin{array}{c}\text { Très } \\
\text { souvent }\end{array}$ & Non & Manuel & Manuel \\
\hline 11 à 15 & $\mathbf{F}$ & $\mathbf{s}$ & Jamais & Oui, Ajust. & Manuel & Guide \\
\hline
\end{tabular}

Nous remarquons tout d'abord que les deux seuls enseignants ayant choisi leur manuel (B et $\mathrm{H}$ ) déclarent le mettre très fréquemment dans les mains de leurs élèves. Ce qui pourrait laisser penser que le fait de choisir le manuel constitue un élément important pour sa fréquence d'utilisation en classe par les élèves. Toutefois, les enseignants E, G et I qui n'ont pas choisi le manuel disent aussi mettre ce support assez ou très souvent dans les mains de leurs élèves.

Pour la conception de leur enseignement, les deux enseignants qui ont choisi leur manuel l'utilisent très différemment puisque l'un des deux $(\mathrm{H})$ suit le guide de l'enseignant et la programmation proposée tandis que l'autre (B) n'utilise pas le guide et ne suit pas la programmation induite par le manuel. Pour ce dernier (B), le manuel semble consister en une banque d'exercices pour les élèves.

Nous notons ensuite que les cinq enseignants de notre étude utilisant le guide de l'enseignant référé au manuel de l'élève présent en salle de classe, que ce soit seul (I) ou avec le manuel de l'élève (A, C, E et $\mathrm{H}$ ), déclarent suivre la programmation prescrite.
Le guide de l'enseignant, quand il est utilisé, semble donc organiser le travail en classe sur l'année.

Par ailleurs, le tableau de la figure 8 montre qu'il existe une diversité d'usage et de mise à disposition du manuel qui n'est pas éclairée par le critère « ancienneté de service » du professeur. Nous trouvons dans les trois tranches d'ancienneté de service (2 à 5 ans, 6 à 10 ans et 11 à 15 ans) des enseignants mettant un manuel entre les mains des élèves de manière plus ou moins fréquente, et des enseignants utilisant le guide seul ou le guide et le manuel pour la conception des séances.

Enfin, notre analyse révèle une grande diversité de liens entre l'usage par le professeur du manuel de l'élève et du guide de l'enseignant et la mise du manuel scolaire entre les mains des élèves. En effet, quatre enseignants $(\mathrm{A}, \mathrm{C}, \mathrm{E}$ et $\mathrm{H})$ déclarent utiliser à la fois le guide du professeur et le manuel de l'élève présent dans la classe pour concevoir leurs séances alors que chacun opte pour une fréquence différente de mise à disposition (Jamais [C], Peu souvent [A], Assez souvent $[E]$, Très souvent $[H]$ ). Cinq autres (B, F, G, I et J) utilisent soit le guide de l'enseignant, soit le manuel de l'élève mais jamais ces deux ouvrages conjointement. Là encore, la fréquence de mise à disposition du manuel entre les mains des élèves varie selon les professeurs (Jamais [F], Peu souvent [J], Très souvent [B, G et I]). Le cas de l'enseignant $\mathrm{D}$ est particulier car il n'utilise ni le guide ni le manuel dont il dispose en classe, que ce soit pour préparer ses séances ou pour donner des exercices aux élèves (qui n'ont donc jamais le manuel en main). Nous pouvons ainsi conclure que, dans notre échantillon, il n'existe pas de lien entre la fréquence de mise à disposition des élèves d'un manuel scolaire et l'utilisation pour la conception de l'enseignement de ce même manuel et/ou du guide associé.

\section{Le rapport au support selon la typologie de Leroyer}

Ces éléments contribuent à pouvoir catégoriser le rapport au support selon la typologie de Leroyer (2013) en utilisateur, adaptateur ou concepteur au regard de leur utilisation des ressources pour la conception de leur enseignement. Mais en appliquant cette grille aux enseignants observés et interviewés, nous nous heurtons à des contradictions. Prenons par exemple le cas du professeur B ; d'une part il 
déclare « s'appuyer régulièrement sur les exercices que propose le manuel », ce qui en ferait un utilisateur, mais d'autre part il déclare "n'utiliser jamais le guide pédagogique " alors que pour la conception des situations de recherche, il « part de vraiment quelque chose de complètement à côté ", par exemple "j'ai fabriqué cette jolie droite graduée », ce qui en ferait un concepteur.

Alors dans quelle catégorie classer le professeur B ? Cette interrogation nous a conduits à un aménagement de l'utilisation de la typologie proposée par Leroyer. Tandis que cette typologie de Leroyer s'applique à la conception de la séance considérée dans sa globalité, nous proposons, tout en gardant les définitions de concepteur, adaptateur, utilisateur fixées par Leroyer, d'affiner l'application de cette typologie en isolant, pour l'analyse, différentes phases structurant la conception. Au regard des éléments qui sont apparus fréquemment dans le discours des enseignants, nous avons retenu, pour cette étude, les entrées suivantes : la programmation annuelle des séances, la situation de départ, les exercices.

Par ailleurs, lorsque les enseignants parlent du rapport qu'ils entretiennent avec les manuels scolaires ou les guides pédagogiques, ils ne considèrent pas uniquement le manuel présent dans la classe et son guide associé mais ils font référence à l'ensemble des ressources dont ils disposent.

Par exemple, l'enseignant $C$, qui rejette totalement le manuel scolaire présent dans la classe, utilise un autre manuel pour établir sa programmation. Il déclare que ce manuel " lui donne un cadre ». En ce sens, nous le classons « Utilisateur » dans l'entrée « Programmation».

Figure 9.

Classification des enseignants lors de différentes phases de la conception de leur enseignement

\begin{tabular}{|l|l|l|l|l|}
\hline $\begin{array}{c}\text { AGS } \\
\text { en années }\end{array}$ & Enseignant & Programmation & $\begin{array}{l}\text { Situation de } \\
\text { départ }\end{array}$ & \multicolumn{1}{|c|}{ Exercice } \\
\hline 2 à 5 & $\mathrm{C}$ & Utilisateur & Adaptateur & Adaptateur \\
\hline 2 à 5 & $\mathrm{E}$ & Adaptateur & Utilisateur & Adaptateur \\
\hline 6 à 10 & $\mathrm{D}$ & Concepteur & Concepteur & Concepteur \\
\hline 6 à 10 & $\mathrm{G}$ & Adaptateur & $\begin{array}{l}\text { Utilisateur / } \\
\text { Concepteur }\end{array}$ & Utilisateur \\
\hline 6 à 10 & $\mathrm{H}$ & Adaptateur & $\begin{array}{l}\text { Utilisateur } \\
\text { Concepteur }\end{array}$ & Utilisateur \\
\hline 6 à 10 & $\mathrm{I}$ & Utilisateur & $\begin{array}{l}\text { Utilisateur } \\
\text { Adaptateur }\end{array}$ & Utilisateur \\
\hline 11 à 15 & $\mathrm{~A}$ & Adaptateur & Adaptateur & Utilisateur \\
\hline 11 à 15 & $\mathrm{~B}$ & Concepteur & Concepteur & Utilisateur \\
\hline 11 à 15 & $\mathrm{~F}$ & Adaptateur & Adaptateur & Adaptateur \\
\hline
\end{tabular}

FIG. 9. - La juxtaposition dans la figure $n^{\circ} 9$ de deux catégories (Utilisateur / Concepteur ou Utilisateur / Adaptateur) signifie que la catégorie des enseignants varie selon la «Situation de départ».
Le tableau de la figure 9 montre que nous trouvons dans les trois tranches d'ancienneté de service (2 à 5 ans, 6 à 10 ans et 11 à 15 ans) des enseignants utilisateur ou adaptateur selon au moins une des trois phases étudiées " programmation », " situation de départ », " exercice ». Notons que les deux enseignants débutants de notre échantillon (classe 2-5 ans) ne se déclarent jamais concepteurs. En outre, dans cette étude qui concerne le niveau CM1, nos résultats, pour les entrées " programmation » et " situation de départ ", semblent rejoindre ceux de Leroyer (2010, p 95) pour qui, dans la tranche des 11-15 ans d'ancienneté et pour le niveau CM2, « le rapport au support » relève « de l'adaptation ou de la conception $»$. Ainsi, le type de rapport que les enseignants entretiennent avec le manuel ou le guide pédagogique varie en fonction de la phase de conception considérée conduisant à des profils différents selon les enseignants.

Contrairement aux études précédentes ciblées sur le public spécifique que sont les professeurs des écoles expérimentés (Margolinas \& Wozniak, 2009a) ou les professeurs des écoles maîtres formateurs (Priolet, 2014), notre enquête s'adresse à des professeurs « ordinaires». Elle permet d'identifier chez deux d'entre eux au moins ( $C$ et I) la présence d'un document principal. Par exemple, $C$ déclare se servir de "Maths Tout terrain » pour « construire ses cours » et I « s'appuyer sur le guide du maître ». Pour les autres enseignants, les données recueillies ne nous permettent pas d'identifier clairement le document "générateur » parmi les documents cités par les enseignants : par exemple $\mathrm{A}$ " prend trois manuels pour construire sa séance », $C$ « cherche pas mal de séances de guides pédagogiques », D se sert plutôt d'Internet « je vais chercher vers "la classe de Mallory ${ }^{30} "$, elle a pas mal de choses qui sont très bien. ». Ce qui ne signifie pas qu'il n'y ait pas de document générateur.

Concernant l'entrée "exercices", nos résultats confirment ceux de Métoudi et Duchauffour (2001). La moitié des enseignants interrogés considèrent le manuel scolaire comme banque de ressources dans ce domaine. Mais à la différence de l'enquête de Métoudi et Duchauffour, dans laquelle les enseignants avaient eux-mêmes choisi leur manuel, notre échantillon ne comporte que deux enseignants ayant effectivement procédé à ce choix. Huit enseignants de CMl sur dix se trouvent ainsi en présence d'un manuel déjà là, qu'il semble parfois difficile de renouveler immé- 
diatement compte tenu des contraintes budgétaires. Ce manuel est alors plutôt considéré comme « outil d'exercisation» (Spallanzani et al., 2001, p. 33). Que le manuel soit « choisi » ou «subi », on constate son attrait comme « exerciseur». Par exemple, l'enseignant I (manuel subi) déclare « qu'un bon manuel, c'est avant tout une bonne banque d'exercices ». Quant à l'enseignant B (manuel choisi), il précise " ce qui nous avait intéressé dans le livre, c'est la batterie d'exercices ». Ce constat rejoint celui de Gueudet et Trouche (2010, p. 58) d'interaction du professeur avec un ensemble de ressources pour préparer sa classe.

La diversité d'utilisation des manuels par les enseignants fait écho à celle constatée par Remillard (2010, p. 208) quand elle dit que « les formes d'engagement des enseignants peuvent amener à des utilisations tout à fait différentes d'une même ressource. ». On constate (Fig. 4) que le manuel scolaire et le guide pédagogique ne suffisent pas et que tous les enseignants de notre échantillon se tournent vers Internet pour trouver d'autres ressources : pour réaliser des jeux de numération ou de calcul, fabriquer des instruments tels que des règles graduées, mais aussi pour préparer les séances d'enseignementapprentissage. Cette diversification des ressources utilisées lors de la conception des séances fait écho à la double acception d'Adler (2010, p. 25) quand elle évoque d'une part, la « réserve » dans laquelle puise le professeur et d'autre part, les apports continuels qui nourrissent son œuvre.

Appliquée à notre panel de dix enseignants, la typologie de Leroyer telle que nous l'avons affinée, ne permet pas de mettre en évidence une diversité éclairée par le critère « ancienneté générale de service des enseignants (AGS).

\section{Utilisation d'un même manuel dans deux classes différentes}

Cette analyse révèle la diversité des ajustements opérés par les enseignants lorsqu'ils sont utilisateurs du guide pédagogique; elle pose la question des enjeux de ces modifications de contenus. Par exemple, l'enseignant $\mathrm{B}$, en proposant une affiche avec une règle déjà graduée par ses soins, en dixièmes et en centièmes, a résolu lui-même le problème qui aurait dû être dévolu aux élèves pour leur permettre d'accéder à des graduations plus fines de la droite.
Cet ajustement a en quelque sorte privé les élèves de la compréhension de la densité de l'ordre des décimaux. La comparaison et l'analyse des deux séances observées sur l'introduction de l'écriture à virgule révèlent comme l'avait constaté Arditi (2011), des profils différents d'enseignants. Ceux-ci procèdent à des ajustements qui renvoient à leur expertise pédagogique : ils reconstruisent par exemple une séance où l'ouverture du manuel livrerait immédiatement la solution à un problème, avant même que les élèves n'aient eu le temps d'en effectuer la recherche. En éludant le guide pédagogique, ils procèdent aussi parfois à une redéfinition de la tâche initialement prévue par les auteurs du manuel, celle-ci pouvant aller jusqu'à conduire à « des problèmes de cohérence » (Arditi, 2011, p. 361).

\section{Profusion des ressources, validation des savoirs et formation des enseignants}

Les dix enseignants de notre échantillon vont télécharger des ressources en ligne, évolution déjà pointée par Bruillard (2010, p. 226), Internet facilitant l'accès à une " gamme de nouveaux possibles ». Ils se tournent plutôt vers les sites de « collègues » pour « voir comment ils procèdent » et ils impriment alors des jeux, des fiches de préparation de séances, délaissant tout ou partie du guide pédagogique associé au manuel que leurs élèves ont parfois à leur disposition. Ces fiches ne s'inscrivent d'ailleurs pas nécessairement en cohérence avec les choix des auteurs du manuel présent dans la classe, ce qui rejoint les constats de Margolinas et Wozniak (2009b, p. 145) et Arditi (2011, p. 361). Cette attitude pose la question de la validation des savoirs recueillis (Bruillard, 2010 , p. 227). On se trouve alors face à une « tension entre deux modèles possibles : d'une part, celui de l'ensemble de ressources largement indépendantes [...] et d'autre part, celui de l'ouvrage organisé autour d'une pensée » (ibid., p. 230).

La diversité de l'offre pose aussi la question de la cohérence, voire de la pertinence de l'approche des notions mathématiques et de leur enseignement, à la fois sur l'année d'utilisation d'un même manuel, et de son guide éventuel, mais aussi sur un temps plus long.

Se pose ainsi la question de leur articulation, au sein d'une collection et entre collections. Certains enseignants prennent ces éléments en compte dans 
le choix du manuel, à l'inverse d'autres qui doivent composer avec d'autres facteurs, y compris d'ordre matériel. Cette question de la continuité des savoirs est encore plus importante pour les élèves qui changent d'école en cours de scolarité.

Concernant le facteur formation, les résultats révèlent une relation étroite entre l'ancienneté de service et les déclarations concernant la place du manuel scolaire de mathématiques dans la formation initiale des professeurs. Ainsi les deux enseignants de la tranche des 2-5 ans d'ancienneté ( $C$ et $E$ ) font référence au manuel «Cap Maths » présenté par les formateurs de l'IUFM, ceux (A, B et F) de la tranche 11-15 ans au manuel «Ermel » présenté en formation. Notons que quatre (A, C, E et F) de ces cinq enseignants utilisent actuellement un guide de l'enseignant pour la conception des séances. Aucun des quatre enseignants de la tranche des 6-10 ans d'ancienneté (D, G, H et I) ne se remémore avoir abordé la question des manuels de mathématiques lors de sa formation initiale. Notons que seuls, deux d'entre eux (H et I) utilisent actuellement un guide de l'enseignant lors de la phase de conception des séances.

La diversité de l'offre actuelle de ressources conduit nécessairement les enseignants à effectuer des choix. On peut se dire que, sans une formation initiale et continue suffisantes éveillant au regard critique nécessaire au choix de ces ressources, les professeurs risquent de ne pas être en mesure d'exercer le contrôle épistémologique nécessaire à la construction de leur documentation. Un constat de manque de formation déjà pointé en 1977 par Priouret.

\section{CONCLUSION}

Tandis que dans les années soixante-dix, Priouret (1977) dégageait le « statut paradoxal » du « livre scolaire », cette ressource largement présente sur le marché de l'édition, mais grandement décriée par ses détracteurs, nous avons cherché à déceler la place accordée de nos jours par les professeurs des écoles au manuel scolaire, dans leur activité enseignante, en considérant selon Amigues (2003) le double aspect lié d'une part à la conception, d'autre part à la mise en œuvre, d'une séance d'enseignement. Nous avons restreint notre champ d'investigation au domaine des mathématiques à l'école élémentaire en France.
La présente étude, centrée sur le niveau d'enseignement CM1, met en évidence une grande diversité de pratiques enseignantes pour ce qui relève de la mise à disposition, dans la classe, de manuels pour les élèves et pour ce qui relève de l'usage des ressources « manuel » ou " guide » pour les enseignants, à la fois dans la conception que dans la mise en œuvre de leurs séances d'enseignement. Et ce dans un contexte, d'une part de "profusion» et de « diversité » d'offre éditoriale de manuels scolaires de mathématiques édités de 2008 à 2015 pour les niveaux scolaires du CP au CM2, et d'autre part d'absence totale de manuels de mathématiques destinés à des classes multi-niveaux alors que dans l'enseignement élémentaire « deux élèves sur cinq sont dans une classe à plusieurs niveaux » (DEPP, 2014).

Dans un contexte qui place les enseignants en situation de choix dans un système éducatif mettant en avant la liberté pédagogique de l'enseignant, nos travaux, en mobilisant le concept de « rapport au support » (Leroyer, 2010), visent à analyser les relations que les professeurs des écoles tissent avec la documentation scolaire, notamment avec le manuel scolaire présent dans leur classe et avec le guide pédagogique qui l'accompagne. Alors que les travaux de Leroyer portent sur le rapport au support, dans la préparation de leur enseignement, de professeurs des écoles de différents âges, avec une ancienneté professionnelle variable et enseignant à différents niveaux de l'école primaire, notre étude vise à analyser le rapport au support des enseignants à la fois dans les phases de conception et dans les phases de mise en ouvre de leurs séances d'enseignement. La méthodologie que nous mettons en œuvre diffère de celle de Leroyer sur deux points : notre approche est strictement qualitative, basée sur l'analyse d'observations de séances in situ complétée par des entretiens de type semi-directif; notre échantillon est constitué de dix professeurs des écoles enseignant au niveau CMl avec une ancienneté de services d'enseignement variant entre 2 et 15 années. Dès lors que nous nous intéressons à des professeurs ordinaires, notre étude vient compléter des travaux existants parfois centrés sur l'usage des manuels par un type de public défini préalablement tels que des professeurs des écoles « expérimentés et sans lien avec la recherche " (Margolinas \& Wozniak, 2009a, p. 65), ou des professeurs des écoles maîtres-formateurs (Priolet, 2014). Étant donné que nous considérons l'ensemble des manuels de mathématiques dispo- 
nibles dans les classes de notre échantillon, elle se démarque aussi de celle de Arditi (2011) qui porte sur l'usage d'un manuel spécifique.

S'agissant du rapport que le professeur entretient avec le manuel scolaire dans la phase de mise en œuvre de son enseignement, nos résultats permettent de mettre en évidence un élément essentiel lié au choix du manuel présent dans la classe et pouvant être mis à portée de main de chacun des élèves. En effet, dans notre échantillon, seulement deux enseignants sur dix ont choisi le manuel de mathématiques présent dans leur classe : ceux-ci déclarent le faire utiliser " très souvent " par leurs élèves. Pour les huit autres enseignants qui se sont retrouvés face à un manuel déjà-là, si trois l'ont adopté et déclarent le faire utiliser « très souvent » ou « assez souvent », deux professeurs déclarent n'en proposer qu'une utilisation «épisodique » en classe tandis que les trois autres n'ont pas mis à portée de main des élèves le manuel, présent dans la classe. Nous pointons là un double paradoxe : le manuel scolaire largement présent sur le marché de l'édition scolaire mais absent pour les classes multi-niveaux, est présent dans la classe mais absent de la main des élèves. Il se trouve mis à distance de ceux à qui il est prioritairement destiné, les élèves. L' « ancienneté de service » n'apparaît pas comme un facteur déterminant pour expliquer la décision des enseignants de mettre ou de ne pas mettre le manuel entre les mains de leurs élèves. Les motifs de rejet total évoqués sont liés au ressenti négatif des enseignants pour lesquels le manuel «ne parle pas » en termes de "format des séances » ou à la non-adaptation de ce support à une classe à cours multiples.

S'agissant maintenant du rapport au support que les professeurs entretiennent avec le manuel scolaire et avec le guide pédagogique dans la phase de conception de leur enseignement, il ressort que neuf professeurs sur les dix se réfèrent soit au manuel présent dans la classe sans nécessairement le mettre à portée de main des élèves, soit au guide pédagogique associé, soit à ces deux ressources simultanément pour "préparer les séances de mathématiques». Mais face à la diversité constatée des types de rapport que les enseignants entretiennent avec le manuel ou le guide pédagogique, il a été nécessaire d'affiner l'application de la typologie de Leroyer (2013) en ne considérant pas l'objet « conception de l'enseignement » en tant qu'entité, mais au contraire, en « cassant » cet objet et en isolant différentes phases de la conception des séances : programmation, situation de départ, exercices. Nous constatons que le rapport au support varie en fonction de la phase de conception de la séance. En effet, pour sept enseignants de notre échantillon, la typologie varie selon les phases de conception. Cependant, au regard du critère " ancienneté générale de service des enseignants », dans cette étude centrée sur le niveau CM1, nos résultats, pour les entrées "programmation» et «situation de départ ", semblent rejoindre ceux de Leroyer (2010, p 95) pour qui, dans la tranche des 11-15 ans d'ancienneté et pour le niveau CM2, « le rapport au support » relève « de l'adaptation ou de la conception $»$.

Notre étude, basée aussi sur l'observation in situ de séances d'enseignement et d'entretiens avec les professeurs, renvoie aussi à la question de la formation des enseignants. Les professeurs de notre échantillon considèrent qu'ils sont peu préparés à l'utilisation du manuel scolaire; selon trois d'entre eux, cette question n'a même jamais été abordée au cours de leur formation initiale ou continue. Les ajustements identifiés lors de l'observation de deux professeurs de CMl en situation d'enseignement de l'introduction de l'écriture à virgule révèlent un problème de cohérence, déjà pointé par Arditi (2011) entre les ajustements opérés par les enseignants-utilisateurs et les auteurs-concepteurs du manuel. En effet, pour l'un des deux professeurs, les ajustements ont conduit à une redéfinition de la tâche prévue par les auteurs du manuel, modifiant ainsi le dispositif didactique prévu, le faisant passer d'un modèle « observation - explication - application » induit par le guide pédagogique, à un modèle du type « explication - application » (Rey, 2001), privant en quelque sorte les élèves de la compréhension de la densité de l'ordre des décimaux. Notons aussi qu'aucun des dix professeurs de notre échantillon n'évoque le contenu didactique des manuels comme critère de choix ou de rejet des manuels, alors qu'au niveau CMl se pose la question de l'apprentissage de notions nouvelles avec notamment l'introduction de l'écriture à virgule. Ces constats renvoient à la question de la validation des savoirs recueillis (Bruillard, 2010) notamment dans un contexte où se développe le recours aux ressources numériques et ainsi au risque d'un manque de contrôle épistémologique lors de la construction de l'enseignement par les professeurs. Ils posent la question de la formation à l'usage des manuels scolaires, des guides pédagogiques et d'une 
façon plus générale, des ressources mobilisées par les professeurs, rejoignant ainsi l'interrogation de Margolinas et Wozniak (2009b, p. 145). En effet, les dix enseignants de notre échantillon déclarent recourir à Internet. Il resterait à identifier le statut des sites en ligne mobilisés, notre étude ne permettant pas de préciser si ces sites prennent chez certains enseignants le statut de « document générateur».

Une étude complémentaire pourrait permettre de poursuivre la réflexion de Leroyer (2010, p. 96) sur « l'impact de la formation institutionnelle et en particulier de la formation initiale » sur le rapport au support des enseignants, notamment dans un contexte de recours par les enseignants à une pluralité de ressources. Il nous semble ainsi que notre étude ouvre la voie à d'autres objectifs de recherche : - dans un contexte d'utilisation de nombreuses ressources notamment en ligne, identifier le document générateur et ses liens avec les autres ressources mobilisées, notamment les ressources en ligne;

- élargir la réflexion engagée sur les concepts d'utilisateur, d'adaptateur et de concepteur, à d'autres éléments de conception de la séance tels que par exemple l'institutionnalisation des savoirs, la place réservée aux traces écrites des élèves. Préciser ces concepts qui sont à mettre en relation à la fois avec les conceptions des enseignants et les ressources qu'ils utilisent ;

- identifier et caractériser les facteurs qui conduisent les enseignants à utiliser, adapter ou concevoir les éléments nécessaires à la préparation et à la mise en ouvre de leur enseignement, en considérant notamment le rôle de la formation initiale et continue.

\section{NOTES}

1. Dans le contexte de la conférence de consensus « Nombres et opérations : premiers apprentissages à l'école primaire » organisée par le CNESCO, en partenariat avec l'Institut français de l'éducation (IFÉ/ENS de Lyon (12 et 13 novembre 2015).

2. Article L912-1-1 - Loi no 2005-380 du 23 avril 2005 art. 48 JORF 24 avril 2005 : «La liberté pédagogique de l'enseignant s'exerce dans le respect des programmes et des instructions du ministre chargé de l'éducation nationale et dans le cadre du projet d'école ou d'établissement avec le conseil et sous le contrôle des membres des corps d'inspection. »

3. Adler (2010) définit trois catégories de ressources (humaines, matérielles et culturelles). "Ressource» est ici assimilé à artefact, au sens défini par Rabardel (1995) : produit de l'activité humaine, élaboré pour s'inscrire dans une activité finalisée.

4. Loi $\mathrm{n}^{0}$ 2013-595 du 8 juillet 2013 d'orientation et de programmation pour la refondation de l'école de la République. Annexe : la programmation des moyens et les orientations de la refondation de l'école de la République.

5. Enquête réalisée par Chaillet (1985) sur le manuel scolaire et son utilisation à l'école élémentaire : auprès de 1735 instituteurs issus de sept départements géographiquement dispersés : Seine-Saint-Denis, Nord, Haut-Rhin, Morbihan, Puy-de-Dôme, Saône-et-Loire et Aude.

6. Bulletin Officiel hors-série $\mathrm{n}^{\circ} 3$ du 19 juin 2008.

7. Bulletin Officiel spécial no 11 du 26 novembre 2015.

8. Bulletin Officiel $n^{\circ} 29$ du 22 juillet 2010 - Définition des compétences à acquérir par les professeurs, documentalistes et conseillers principaux d'éducation pour l'exercice de leur métier. (Arrêté du 12-5-2010 - J.O. du 18-7-2010). 9. Dans « Concevoir et mettre en ouvre son enseignement / Attitudes ».

10. Bulletin Officiel no 30 du 25 juillet 2013 : Référentiel des compétences professionnelles des métiers du professorat et de l'éducation (Arrêté du 1-7-2013 - J.O. du 18-7-2013).

11. Inventaire qui ne saurait être exhaustif en raison de la profusion de l'offre.

12. 122 titres composent notre recueil de données (en comptant séparément Fichier et Livre).

13. Note d'information $n^{\circ} 44$ : Les élèves du premier degré à la rentrée 2015 : forte baisse des taux de redoublement (DEPP, novembre 2015, p. 4) et données complémentaires (tableaux et graphiques au format Excel).

14. En référence à la scolarité en cycles créé le 10 juillet 1989 par la loi d'orientation sur l'éducation et modifié par le décret du 24 juillet 2013. 
15. Il s'agit du titre de la thèse de Jacques Priouret. Il est à noter également que le "livre scolaire" analysé dans cette thèse inclut tous les livres à destination de l'élève : le « manuel scolaire» mais aussi le « roman scolaire».

16. Pour le Québec, il s'agit des enfants âgés de 10 et 11 ans.

17. Mais qui ne sont pas en fin de carrière.

18. Le document générateur peut par exemple être un guide pédagogique, un manuel, un référentiel de compétences, un document extrait d'un site Internet...

19. On entend ici par professeurs ordinaires, les enseignants qui ne sont pas professeurs des écoles maîtres formateurs. 20.5 professeurs chargés du seul niveau CMl et 5 professeurs chargés d'une classe à double-niveau : CEl-CM1 (1 enseignant) ; CE2-CM1 (2 enseignants) et CM1-CM2 (2 enseignants).

21. Dans cette classe E, le deuxième manuel a été acheté à raison d'un pour deux élèves.

22. Programmes en vigueur de 2008 à 2015.

23. Toute cette mise à distance du manuel pour les élèves n'exclut pas une utilisation par l'enseignant lors de la conception des séances.

24. C'est-à-dire comment ils transforment l'outil « manuel» en instrument. Rabardel (1995) parle de « genèse instrumentale ».

25. Qui correspond à la durée entre l'instant où l'enseignant a demandé aux élèves d'« ouvrir le manuel » et l'instant où il signale la fin d'activité avec le manuel.

26. Avant-propos, Guide pédagogique, À portée de Maths - CM1, p. 3.

27. Selon Rey, les trois types de dispositifs didactiques sont : «explication-application », « observation-compréhension-application » et " problème-compréhension-application» (Rey, 2001, p. 31-35).

28. «À portée de Maths CM1 ».

29. Il faut rappeler que tous les enseignants utilisent Internet et que seuls les enseignants $\mathrm{F}$ et J n'utilisent pas d'autres ressources (jeux mathématiques, logiciels, etc.).

30. La classe de Mallory. Ressources et Jeux Cycle 3. [http://www.laclassedemallory.com/] consulté le 25 septembre 2016. Une étude plus approfondie du statut de cette ressource en ligne permettrait peut-être de lui conférer le statut de document générateur.

\section{RÉFÉRENCES}

Adler, J., Reed, Y., Lelliott, T., \& Setati, M. (2002). Availability and use of resources: a dual challenge for teacher education. Dans J. Adler \& Y. Reed (éds.), Challenges of teacher development. An investigation of take-up in South Africa (p. 53-71). Pretoria : Van Schaik.

Adler, J. (2010). La conceptualisation des ressources. Apports pour la formation des professeurs de mathématiques. Dans G. Gueudet \& L. Trouche, Ressources vives. Le travail documentaire des professeurs en mathématiques (p. 23-39). Rennes: Presses universitaires de Rennes.

Amigues, R. (2003). Pour une approche ergonomique de l'activité enseignante. Skholê, HS 1, 5-16.

Arditi, S. (2011). Variabilité des pratiques effectives des professeurs des écoles utilisant un même manuel écrit par des didacticiens (Thèse de doctorat). Université ParisDiderot-Paris 7, Paris.

Bardin, L. (2007). L'analyse de contenu. Paris : PUF.

Bruillard, E. (2010). Le passage du papier au numérique : le cas du manuel scolaire. Dans G. Gueudet \& L. Trouche, Ressources vives. Le travail documentaire des professeurs en mathématiques (p. 217-232). Rennes: Presses universitaires de Rennes.

Caritey, C. (1993). Manuels scolaires et mémoire historique au Québec. Questions de méthode. Histoire de l'éducation, 58, 137-164.

Chaillet, D. (1985). Le manuel scolaire à l'école élémentaire. Compte-rendu d'enquête. Paris : Ministère de l'Éducation nationale.

Choppin, A. (1980). Lhistoire des manuels scolaires : une approche globale. Histoire de l'éducation, 58, 37-164.

Condorcet (de), N. (1792). Rapport et projet de décret relatifs à l'organisation générale de l'instruction publique. Présentation à l'Assemblée législative. Paris : Assemblée nationale. [http://www.assemblee-nationale. fr/histoire/7ed.asp].

Direction de l'évaluation, de la prospective et de la performance (2014). Note d'information $n^{\circ} 41$. Paris : MEN

Freinet, C. (1928). Plus de manuels scolaires. St Paul : Éditions de l'Imprimerie à l'École.

Gueudet, G., \& Trouche, L. (2010). Des ressources aux documents, travail du professeur et genèse documentaire. Dans G Gueudet \& L. Trouche, Ressources vives. Le travail documentaire des professeurs en mathématiques (p. 57-74). Rennes: Presses universitaires de Rennes.

Goigoux, R. (2002). Analyser l'activité d'enseignement de la lecture : une monographie. Revue française de pédagogie, 138, 125-134.

Goigoux, R. (2007). Un modèle d'analyse de l'activité des enseignants. Éducation \& Didactique, 1(3), 47-70

Goigoux, R., Ria, L., \& Toczek-Capelle, M.-C. (2010). Mieux connaître les parcours de formation des enseignants débutants pour mieux les former. Dans R. Goigoux, L. Ria, M.-C. Toczek-Capelle, Les parcours de formation des enseignants débutants (p. 25-44). 
Clermont-Ferrand : Presses universitaires de Blaise Pascal.

Inspection générale de l'Éducation nationale (1998). Le manuel scolaire. Paris : La Documentation française.

Inspection générale de l'Éducation nationale, Inspection générale de l'administration de l'Éducation nationale et de la Recherche (2010). Le manuel scolaire à l'heure du numérique. Une "nouvelle donne " de la politique des ressources pour l'enseignement. Paris : Ministère de l'Éducation nationale, de la jeunesse et de la vie associative.

Inspection générale de l'Éducation nationale (2012). Les manuels scolaires : situation et perspectives. Paris : Ministère de l'Éducation nationale.

Lebrun, J. (dir.) (2006). Le manuel scolaire, un outil à multiples facettes. Québec : Presses de l'université du Québec.

Lenoir, Y., Hasni, A., Lebrun, J., Larose, F., Maubant, P., Lisée, V., Oliveira, A. A., \& Routhier, S. (2007). Lutilisation des manuels scolaires dans les écoles primaires du Québec : résultats de quinze ans de recherche. Dans M. Lebrun (dir.), Le manuel scolaire d'ici et d'ailleurs, d'hier à demain. (Livre + CD-ROM). Québec: Presses de l'université du Québec.

Leroyer, L. (2010). Supports d'enseignement et préparation de classe. Éducation et Formation, e-292, 83-96.

Leroyer, L. (2013). Le rapport au travail de préparation en mathématiques des enseignants du premier degré. Éducation \& Didactique, 7(1), 147-164.

Margolinas, C., \& Wozniak, F. (2009a). Usage des manuels dans le travail de l'enseignant : l'enseignement des mathématiques à l'école primaire. Revue des sciences de l'éducation, 35(2), 59-82.

Margolinas, C., \& Wozniak, F. (2009b). Place des documents dans l'élaboration d'un enseignement de mathématiques à l'école primaire. Dans I. Bloch \& F. Conne (dir.), Nouvelles perspectives en didactique des mathématiques. La géométrie, les documents pour l'enseignement, le métier de chercheur en didactique. Cours de la XIVe école d'été de didactique des mathématiques (p. 135-146). Grenoble: La pensée sauvage.

Métoudi, M., \& Duchauffour, H. (2001). Des manuels et des maittres. Paris : Belin.

Mollier, J.-Y. (2002). Les maîtres des manuels scolaires. Dans J.-P. Rioux (éd.), Deux cents ans d'Inspection générale 1802-2002 (p. 125-141). Paris : Fayard.
Mounier, E., \& Priolet, M. (2015). Les manuels scolaires de mathématiques à l'école primaire - De l'analyse descriptive de l'offre éditoriale à son utilisation en classe élémentaire. Rapport présenté lors de la conférence de consensus. Nombres et opérations : premiers apprentissages à l'école primaire. Paris : CNESCO, Lyon : IFÉ-ENS.

Nicol, C. C., \& Crespo, S. M. (2006). Learning to teach with mathematics textbooks: How preservice teachers interpret and use curriculum materials. Educational studies in mathematics, 62(3), 331-355

.Priolet, M. (2014). The use of mathematics textbooks in the training of elementary school teachers in France. History of Education et Children's Literature, IX/1, 111-124.

Priouret, J. (1977). Réflexion sur le statut paradoxal du livre scolaire (Thèse de doctorat d'État). Université Lyon 2, Lyon.

Rabardel, P. (1995). Les hommes et les technologies; approche cognitive des instruments contemporains. Paris : Armand Colin.

Remillard, J. (2010). Modes d'engagement : comprendre les transactions des professeurs avec les ressources curriculaires en mathématiques. Dans G. Gueudet \& L. Trouche, Ressources vives. Le travail documentaire des professeurs en mathématiques (p. 201-216). Rennes: Presses universitaires de Rennes.

Rey, B. (2001). Manuels scolaires et dispositifs didactiques. Dans Y. Lenoir, B. Rey, G.-R. Roy \& J. Lebrun (éd.), Le manuel scolaire et l'intervention éducative: regards critiques sur ses apports et ses limites (p. 25-40). Sherbrooke : Éditions du CRP.

Sensevy, G. (2011). Le sens du savoir : éléments pour une théorie de l'action conjointe en didactique. Bruxelles : De Boeck.

Spallanzani, C., Biron, D., Larose, F., Lebrun, J. (dir.), Lenoir, Y., Masselter, G. (dir.), \& Roy, G.-R. (2001). Le rôle du manuel scolaire dans les pratiques enseignantes au primaire, Sherbrooke : Éditions du CRP.

Theureau, J. (2010). Les entretiens d'autoconfrontation et de remise en situation par les traces matérielles et le programme de recherche « cours d'action ». Revue d'anthropologie des connaissances, 4(2), 287-322. 


\section{ANNEXE 1 : OFFRE ÉDITORIALE RECENSÉE (MARS 2015)}

FIG. 10. - F : Fichier / L : Livre

\begin{tabular}{|c|c|c|c|c|c|c|c|}
\hline Éditeur & Titre & $\begin{array}{c}\text { Dispo } \\
\text { Rentrée } \\
2014 \\
\end{array}$ & $\mathrm{CP}$ & CE1 & CE2 & CM1 & $\mathrm{CM} 2$ \\
\hline Belin & La clé des maths & 6 & $\mathrm{~F}$ & $\mathrm{~F}$ & $\mathrm{FL}$ & $\mathrm{L}$ & $\mathrm{L}$ \\
\hline Belin & J'aime les maths & 3 & $\mathrm{~F}$ & $\mathrm{~F}$ & $\mathrm{~F}$ & & \\
\hline Bordas & Au rythme des maths & 5 & $\mathrm{~F}$ & & $\mathrm{FL}$ & $\mathrm{L}$ & $\mathrm{L}$ \\
\hline Bordas & Maths tout terrain & 8 & $\mathrm{~F}$ & $\mathrm{~F}$ & $\mathrm{FL}$ & $\mathrm{FL}$ & $\mathrm{FL}$ \\
\hline Bordas & Maths collection Thévenet + & 6 & $\mathrm{~F}$ & $\mathrm{~F}$ & $\mathrm{FL}$ & $\mathrm{L}$ & $\mathrm{L}$ \\
\hline Bordas & Place aux maths & 2 & $\mathrm{~F}$ & $\mathrm{~F}$ & & & \\
\hline Celda & Act Mathématiques & 3 & & & $\mathrm{~F}$ & $\mathrm{~F}$ & $\mathrm{~F}$ \\
\hline Hachette & A portée de Maths & 4 & & $\mathrm{~L}$ & $\mathrm{~L}$ & $\mathrm{~L}$ & $\mathrm{~L}$ \\
\hline Hachette & $\begin{array}{l}\text { Les maths à la découverte } d u \\
\text { monde / des sciences }\end{array}$ & 4 & $\mathrm{~F}$ & $\mathrm{~F}$ & $\mathrm{~F}$ & & $\mathrm{~L}$ \\
\hline Hachette & Mathématiques Petit Phare & 3 & & & $\mathrm{~F}$ & $\mathrm{~L}$ & $\mathrm{~L}$ \\
\hline Hachette & Pour comprendre les maths & 7 & $\mathrm{~F}$ & $\mathrm{~F}$ & $\mathrm{FL}$ & $\mathrm{FL}$ & $\mathrm{L}$ \\
\hline Hachette & Maths Explicites & 1 & & & & $\mathrm{~L}$ & \\
\hline Hatier & Cap Maths & 6 & $\mathrm{~F}$ & $\mathrm{~F}$ & $\mathrm{FL}$ & $\mathrm{L}$ & $\mathrm{L}$ \\
\hline Hatier & Euro Maths & 6 & $\mathrm{~F}$ & $\mathrm{~F}$ & $\mathrm{FL}$ & $\mathrm{L}$ & $\mathrm{L}$ \\
\hline Istra & Mathématiques Litchi & 6 & $\mathrm{~F}$ & $\mathrm{~F}$ & $\mathrm{FL}$ & $\mathrm{L}$ & $\mathrm{L}$ \\
\hline $\begin{array}{l}\text { La librairie } \\
\text { des écoles }\end{array}$ & Méthode de Singapour & 5 & $\mathrm{~F}$ & $\mathrm{~F}$ & $\mathrm{~F}$ & $\mathrm{~F}$ & $\mathrm{~F}$ \\
\hline Magnard & La tribu des Maths & 5 & $\mathrm{~F}$ & $\mathrm{~F}$ & $\mathrm{~L}$ & $\mathrm{~L}$ & $\mathrm{~L}$ \\
\hline Magnard & $\begin{array}{l}\text { Mes premières } \\
\text { mathématiques avec Nemo et } \\
\text { Mila }\end{array}$ & 1 & $\mathrm{~F}$ & & & & \\
\hline Magnard & Outils pour les maths & 7 & $\mathrm{~F}$ & $\mathrm{FL}$ & $\mathrm{FL}$ & $\mathrm{L}$ & $\mathrm{L}$ \\
\hline Nathan & Tous en Maths & 5 & $\mathrm{~F}$ & $\mathrm{~F}$ & $\mathrm{FL}$ & $\mathrm{L}$ & \\
\hline Nathan & Vivre les maths & 8 & $\mathrm{~F}$ & $\mathrm{~F}$ & $\mathrm{FL}$ & $\mathrm{FL}$ & $\mathrm{FL}$ \\
\hline Retz & $\begin{array}{l}\text { J'apprends les Maths avec } \\
\text { Picbille }\end{array}$ & 1 & $\mathrm{~F}$ & & & & \\
\hline Retz & $\begin{array}{l}\text { J'apprends les Maths avec } \\
\text { Tchou }\end{array}$ & 1 & $\mathrm{~F}$ & & & & \\
\hline Retz & J'apprends les Maths & 5 & & $\mathrm{~F}$ & $\mathrm{FL}$ & $\mathrm{L}$ & $\mathrm{L}$ \\
\hline Retz & Les cahiers Sésamath & 1 & & & & & $\mathrm{~F}$ \\
\hline Sed & Classe Maths & 3 & $\mathrm{~F}$ & $\mathrm{~F}$ & $\mathrm{~F}$ & & \\
\hline Sed & Maths + & 5 & $\mathrm{~F}$ & $\mathrm{~F}$ & $\mathrm{~L}$ & $\mathrm{~L}$ & $\mathrm{~L}$ \\
\hline \multirow[t]{2}{*}{ Sedrap } & Compagnon Maths & 5 & $\mathrm{~F}$ & $\mathrm{~F}$ & $\mathrm{~L}$ & $\mathrm{~L}$ & $\mathrm{~L}$ \\
\hline & & 122 & 22 & 21 & 34 & 23 & 22 \\
\hline
\end{tabular}

Note : Les manuels « J'apprends les Maths avec Picbille » et « J'apprends les Maths avec Tchou » ont été considérés comme des variantes de la collection « J'apprends les Maths ». Les 7 manuels sont donc considérés comme faisant partie de la même collection.

Le fichier se différencie du livre en se présentant sous l'aspect d'un cahier personnalisé sur lequel l'élève écrit les réponses aux exercices. 